\title{
ON SECONDARY BIFURCATIONS FOR SOME NONLINEAR CONVOLUTION EQUATIONS ${ }^{1}$
}

\author{
F. COMETS, TH. EISELE AND M. SCHATZMAN
}

\begin{abstract}
On the $d$-dimensional torus $\mathbf{T}^{d}=(\mathbf{R} / \mathbf{Z})^{d}$, we study the nonlinear convolution equation

$$
u(t)=g\{\lambda \cdot w * u(t)\}, \quad t \in \mathbf{T}^{d}, \lambda>0 .
$$

where * is the convolution on $\mathbf{T}^{d}, w$ is an integrable function which is not assumed to be even, and $g$ is bounded, odd, increasing, and concave on $\mathbf{R}^{+}$. A typical example is $g=$ th.

For a general function $w$, we show by the standard theory of local bifurcation that, if the eigenspace of the linearized problem is of dimension 2 , a branch of solutions bifurcates at $\lambda=\left(g^{\prime}(0) \hat{w}(p)\right)^{-1}$ from the zero solution, and we show that it can be extended to infinity.

For special simple forms of $w$, we show that the first bifurcating branch has no secondary bifurcation, but the other branches can.

These results are related to the theory of spin models on $\mathbf{T}^{d}$ in statistical mechanics, where they allow one to show the existence of a secondary phase transition of first order, and to some models of periodic structures in the brain in neurophysiology.
\end{abstract}

1. Introduction. The aim of this paper is to analyse the branches of solutions of a nonlinear convolution equation on the $d$-dimensional torus $\mathbf{T}^{d}=(\mathbf{R} / \mathbf{Z})^{d}$. The equations are of the general form

$$
u(t)=g\{\lambda w * u(t)\},
$$

where $t \in \mathbf{T}^{d}, \lambda \in \mathbf{R}_{+}$, * is the convolution operator, $w$ a given integrable function on $\mathbf{T}^{d}$, which is not assumed to be even, and $g$ is a bounded, odd, increasing function, which is concave on $\mathbf{R}^{+}$. The positivity of $\lambda$ does not reduce the generality.

There is a large number of models where equations of the above kind appear, in particular within the theory of statistical mechanics and some mathematical models of biology. In statistical mechanics, (1.1) corresponds to the mean field equation of an interacting spin system (see $[\mathbf{2 0}, \mathbf{2}]$ ). In the thermodynamical limit, the free energy $\psi(\beta)$ of the system is given by a variational principle

$$
-\beta \psi(\beta)=\sup _{u \in L_{2}\left(\mathbf{T}^{d}\right)}\left[\beta \iint_{\left(\mathbf{T}^{d}\right)^{2}} w(t-s) u(s) u(t) d s d t-\int_{\mathbf{T}^{d}} i_{\rho}(u(t)) d t\right],
$$

Received by the editors June 4, 1984.

1980 Mathematics Subject Classification (1985 Revision). Primary 45G10, 47H15, 45E10; Secondary 60K35, 82A25, 92A09.

1 This work has been supported by the Deutsche Forschungsgemeinschaft. 
where $w(t-s)$ represents the interaction potential between a spin at site $t$ and a spin at site $s, w$ is assumed to be even, but not necessarily positive, and $i_{\rho}$ is the entropy function of the single spin distribution $\rho$ :

$$
i_{\rho}(x)=\sup _{y}\left\{x y-\ln \int \exp (y z) \rho(d z)\right\}
$$

(see also §2). If $u_{0}$ is a (local) maximum of the variational problem (1.2), then the first Fréchet derivative of $\beta F-I$ must vanish; i.e.,

$$
\beta F^{\prime}\left(u_{0}\right)-I^{\prime}\left(u_{0}\right)=0,
$$

or equivalently,

$$
\beta w * u_{0}(t)-i_{\rho}^{\prime}\left(u_{0}(t)\right)=0
$$

almost everywhere. This mean field equation is equivalent to (1.1) if we set $i_{\rho}^{\prime}=g^{-1}$ and replace the inverse temperature $\beta$ by the parameter $\lambda$. The global maxima of (1.2) correspond to equilibrium states, while local maxima represent metastable states. Both are stable solutions of (1.5) or (1.1) (see §6). Moreover, in the theory of nucleation (see $[\mathbf{1 5}, \mathbf{2 3}]$ ), one is interested in solutions of $(1.5)$ which are saddle points of the potential $\beta F-I$. They are unstable, or more precisely hyperbolic, solutions of (1.5) in the sense of dynamical systems.

Phase transitions of the spin system are nonanalytic changes of the global maximum $u_{0}$ of the variational principle. They are in general linked with a bifurcation of the solutions of the mean field equation (1.5) and simultaneously with a change of the stability of the solutions of (1.5). In [2] it has been shown that there are primary stable bifurcations of the solutions of (1.1) not only for the nonzero constant solutions (Curie-Weiss model), but also for periodic solutions of (1.1), which appear in the antiferromagnetic case.

Beside these models, in which equation (1.1) appears literally, there are a number of models where one gets equations of a similar type. We like to refer especially to the spin-glass model of van Hemmen et al. [10], since in particular one studies there secondary phase transitions - corresponding to secondary bifurcations of (1.1) here - which establish the existence of so-called mixed phases. The similarity of the equations mentioned in this reference and (1.1) will become even more obvious after we have transformed (1.1) into the corresponding equations for the Fourier transforms in $\S 2$. The methods developed in this paper, and in particular those of the associated dynamical system $(\S 8)$ allow one to understand better the results of [10].

Nonlinear evolution equations involving a convolution term appear also in some mathematical models of biological systems. We shall mention $[\mathbf{0}, \mathbf{1}, \mathbf{5}, \mathbf{6}$, and 16], where further references are quoted, but let us give more details about the problems addressed in [16] because they are the closest to the ones we consider here.

The adult brain of higher organisms such as mammals displays a remarkable mixture of highly specific connectivity patterns with large amounts of randomness. The cortex is the external part of the brain; it is an envelope about $2 \mathrm{~mm}$ thick, with many folds. The visual cortex, which has been extensively studied, is located in the occipital region, and it receives indirect projections from the two retinae. The existence of ocular dominance stripes is among the striking organization patterns uncovered in the sixties: in the brain of adult animals, the cells are segregated into 
stripes which are sensitive either to left eye or to right eye stimuli; but this is not true in newborn animals.

It is a major problem to understand the rules which guide the formation of this circuitry during pre- and postnatal development. A theoretical explanation should show how microscopic mechanisms governing the growth and decay of synapsesthe individual contacts between neurones-yield the observed macroscopic behavior. Models of development of ocular dominance stripes stipulate that growth of contacts at points $x$ depends on the density of fibres or contacts not only at $x$, but in a neighborhood of $x$.

Two alternative types of mechanisms may be invoked. In the first, afferent fibres carry chemical markers which diffuse laterally within the cortical tissue; at point $x$ in cortex, the rate of growth of synapses of a certain type-i.e., coming from either the left eye of the right eye-is governed by the similarity between the marker carried by the fibre, and the concentration of this marker at $x[\mathbf{1 3}]$. In the second type of model, synaptic growth depends solely on short-term temporal correlations between pre- and postsynaptic activities: this is an application of the Hebb principle of synaptic modification [8]. According to this principle, the strength of connections between two cells grows proportionally to the correlation between the activities of the two cells. Activities in fibres of different origins-right and left eye-are assumed to be uncorrelated, and correlations or anticorrelations are carried through the cortex via a pre-existing circuitry [22].

It has been pointed out [18] that, in spite of different mechanisms, the two models are theoretically equivalent; both are conveniently summarized by an evolution equation with a spatial convolution term of a particular type: the central part of the convolution kernel is positive, the outer part negative. If the variable $u$ designates the difference between the density of left-eye and right-eye contacts, the evolution of $u$ is described by the following equation [18], where $w$ is a given convolution kernel depending only on space and $*$ is the spatial convolution:

$$
\partial u / \partial t=(w * u) \cdot f(u)
$$

The nonlinearity $f$ serves to express a saturation or constraint; a modification of this equation, which has the advantage of exhibiting better the effect of the constraints, if for instance there is a physically maximal density of contacts, is

$$
\partial u / \partial t=w * u-h(u)
$$

where $h$ is an increasing function of $u$, which can be taken multivalued if sharp constraints are desired. We would like to study the behavior of (1.6) and (1.7), as time increases infinitely.

It is shown in [16] that the nontrivial stable solutions of $(1.6)$ when $f(u)=1-u^{2}$ satisfy, under a suitable functional hypothesis on $w$,

$$
u=\operatorname{sgn}(w * u)
$$

and that the nontrivial stable stationary solutions of (1.7) satisfy

$$
h(u)=w * u .
$$

Clearly, if $g$ is the reciprocal of the signum function, which means that, in (1.7), $u$ is constrained to stay between -1 and +1 , problems (1.8) and (1.9) are identical. 
If we write (1.9) as

$$
u=h^{-1}(w * u),
$$

it is natural to imbed (1.10) in a family of similar problems depending on a parameter $u=h^{-1}(\lambda w * u)$, which is precisely problem (1.1) considered above in a statistical physics setting. If, in particular, we take $g=h^{-1}=$ th as in (2.10), we obtain

$$
u=\operatorname{th}(\lambda w * u) \text {. }
$$

Observe that as $\lambda$ goes to infinity, problem (1.11) resembles more and more problem (1.8). We expect to gain some understanding of problems (1.6) and (1.7) through a careful study of the set of their stable stationary solutions, which are the main candidates to be asymptotic states of (1.6) and (1.7) as time grows infinitely. Thus we are interested in a rather complete description of all solutions of (1.1), at least for some natural choices of the function $w$.

This paper contains

(a) The proof that if $\lambda \in\left(0,\left(g^{\prime}(0) \widehat{|w|}(0)\right)^{-1}\right)$, the only solution of $(1.1)$ is zero. Here and below, $\hat{f}(p)=\int_{\mathbf{T}^{d}} f(t) \exp \{-2 \pi i p t\} d t$ denotes the Fourier coefficient of the function $f$ on $\mathbf{T}^{d}, p \in \mathbf{Z}^{d}$.

(b) A description of the primary bifurcation picture. Assuming $\hat{w}(p)$ real and $\hat{w}(q) \neq \hat{w}(p)$ for all $q \neq \pm p$, we obtain in some cases a branch starting at $\lambda_{p}=$ $\left(g^{\prime}(0) \hat{w}(p)\right)^{-1}$ and extending to infinity. We do not presently cover the cases when $w$ has symmetries in $\mathbf{T}^{d}, d>1$, i.e., $\hat{w}(p)=\hat{w}(q)$ for some $q \neq \pm p$, because this would lead to bifurcation kernels of dimension larger than 2 .

(c) A description of secondary bifurcations for some special choices of $w$. More precisely, if we assume that

$$
w(t)=\alpha \cos (2 \pi p t)+\beta \cos (2 \pi q t)+w_{0}(t)
$$

with $\alpha, \beta>0$

$$
\hat{w}_{0}(r)=0 \text { for } r \in[(2 \mathbf{Z}+1) p+2 \mathbf{Z} q] \cup[2 \mathbf{Z} p+(2 \mathbf{Z}+1) q],
$$

and $p, q$ satisfying either the noncollinearity condition

$$
p=0 \neq q \quad \text { or } \quad(p p)(q q)-(p q)^{2}>0,
$$

or in the collinear case, the arithmetic condition

$$
q \notin(2 \mathbf{Z}+1) p \text { and } p \notin(2 \mathbf{Z}+1) q
$$

then we are able to give a rather complete picture of the secondary bifurcations in Theorems 5 and 7 . In particular, no second bifurcation from the first-appearing branch occurs, but some may occur from the second branch. This secondary bifurcation is connected with an exchange in the stability of the primary branch. In the noncollinear case, this branch is unstable, or more precisely hyperbolic (see $\S 8$ for the definition), until the appearance of the secondary bifurcation, but it is stable after the occurrence of the secondary bifurcation. The solutions on the secondary branch are, in general, hyperbolic.

In an example we show that the mentioned exchange in stability on the second of the primary branches, which goes together with the secondary bifurcation, is 
of physical relevance in some models of statistical mechanics. It gives rise to a secondary phase transition of first order, where the equilibrium state jumps from the first primary branch to another stable solution.

The stability analysis of the different branches is done by reducing the problem to a finite-dimensional one on the Fourier coefficients $\hat{u}( \pm p)$ and $\hat{u}( \pm q)$ and by studying the geometric properties of an associated mapping. When the noncollinearity condition (1.13) holds, the set of solutions can be completely described. Moreover, in this case we characterize the fixed points as stable, hyperbolic, or totally unstable.

Of course, these results depend heavily on the oddness of $g$. Small perturbations from this condition would lead to nonconnected manifolds of solutions, which show turning points and so-called two-sided bifurcations. They appear, for example, in the spin model of the beginning of this section if there exists an additional external magnetic field $h=h(s)$.

Also higher-dimensional spin variables may be treated similarly: for example, $X-Y$ spins or Heisenberg spins with values in $S^{2}$. Their mean field equations have the form of systems of nonlinear convolution equations. However, these generalizations will not be discussed in this paper.

2. General assumptions and preliminary results. Let $\mathbf{T}^{d}=(\mathbf{R} / \mathbf{Z})^{d}$ be the $d$-dimensional torus. By $d t$ we denote the Lebesgue measure on $\mathbf{T}^{d}$. We are concerned with the nonlinear convolution equation

$$
u(t)=g\{\lambda \cdot w * u(t)\}, \quad t \in \mathbf{T}^{d},
$$

where $\lambda \in \mathbf{R}_{+}=[0,+\infty)$. Here, for a given Lebesgue-integrable function $w$ on $\mathbf{T}^{d}$, we define the convolution operator

$$
w * u(t)=\int_{\mathbf{T}^{d}} w(t-s) u(s) d s
$$

and we assume $g: \mathbf{R} \rightarrow \mathbf{R}$ to be an odd, increasing, bounded function which is

$$
\text { concave on }[0, \infty) \text {. }
$$

Here and in the rest of the paper, we understand increasing, decreasing, etc., in the weak sense of nondecreasing, nonincreasing, etc., respectively, and similarly for concave. Otherwise, we say strictly increasing, strictly decreasing, strictly concave, etc. Of course, we exclude the trivial cases $g \equiv 0$ or $w \equiv 0$.

REMARK. In principle, there is no restriction in having $\lambda \geq 0$ instead of $\lambda \in \mathbf{R}$, since the pair $(-\lambda, w)$ gives the same equation (2.1) as the pair $(\lambda,-w)$. However, the formulation of the theorems is much simplified by considering only $\lambda \in \mathbf{R}_{+}$.

In the examples from statistical mechanics, the interaction potential is given by the function $w$, and a thermodynamical state $u$ on $\mathbf{T}^{d}$ has internal energy

$$
E=\frac{1}{2}\langle u, w * u\rangle=\frac{1}{2} \int_{\mathbf{T}^{d}} u(t) \cdot w * u(t) d t .
$$

On the other hand, the nonlinear function $g$ reflects in some sense the entropy of the system. To be more precise, let us recall (see $[\mathbf{1 2}, \mathbf{2}]$, e.g.) the definition of the $\phi$-function for a measure $\rho$ :

$$
\phi_{\rho}(x)=\ln \int \exp (x y) \rho(d y) .
$$


The entropy function $i_{\rho}$ of $\rho$ can then be calculated as the Legendre transformation of $\phi_{\rho}$ :

$$
i_{\rho}(y)=\sup _{x}\left\{x y-\phi_{\rho}(x)\right\} .
$$

Now, $g$ is the derivative of the function $\phi_{\rho}$ or, equivalently, by (2.6), the inverse function of the derivative of the entropy $i_{\rho}$ :

$$
g(x)=\phi_{\rho}^{\prime}(x)=\left(i_{\rho}^{\prime}\right)^{-1}(x)
$$

In examples with Ising spins, we have

$$
\rho_{0}=\left(\delta_{+1}+\delta_{-1}\right) / 2
$$

such that

$$
\phi_{\rho_{0}}(x)=\ln \cosh (x)
$$

and

$$
g_{0}(x)=\phi_{\rho_{0}}^{\prime}(x)=\operatorname{th}(x) .
$$

Obviously, $g_{0}$ satisfies the desired properties (2.3). It is even real analytic on $\mathbf{R}$ and strictly concave on $(0, \infty)$. In general, the concavity condition for $g$ is tantamount to the GHS-inequality for the measure $\rho$ (see $[\mathbf{4}, \mathbf{2}]$ ).

For a one-dimensional problem and in connection with a quadratic internal energy, this inequality guarantees that there is exactly one higher-order phase transition for the equilibrium state (see also [3], in particular the remark at the end of $\S 5)$.

We note some simple consequences from our assumptions on $g: g$ being odd, we have

$$
g(0)=0 .
$$

Because $g$ does not vanish identically and is concave on $\mathbf{R}^{+}$, we find for $x \neq 0$ that

$$
g(x) / x \text { is strictly positive and decreasing with respect to }|x| \text {. }
$$

Therefore,

$$
g^{\prime}(0):=\lim _{|x| \rightarrow 0} \frac{g(x)}{x}>0
$$

exists and is positive. Until $\S 4$ inclusively, we allow $g^{\prime}(0)=+\infty$. Set

$$
\gamma=\lim _{x \rightarrow+\infty} g(x) \in(0, \infty) .
$$

By the concavity condition, $g$ is necessarily continuous on $\mathbf{R} \backslash\{0\}$, possibly with two symmetric jumps at zero. Finally, we set

$$
\bar{g}^{\prime}(x)=\limsup _{|\varepsilon| \rightarrow 0} \frac{g(x+\varepsilon)-g(x)}{\varepsilon} \geq 0,
$$

and

$$
\underline{g}^{\prime}(x)=\liminf _{|\varepsilon| \rightarrow 0} \frac{g(x+\varepsilon)-g(x)}{\varepsilon} \geq 0
$$


$\bar{g}^{\prime}$ and $\underline{g}^{\prime}$ are symmetric, decreasing in $|x|$, and

$$
g^{\prime}(0)=\bar{g}^{\prime}(0)=\underline{g}^{\prime}(0) \text {. }
$$

We mention in particular, that if $g$ is strictly concave on $(0, \infty)$ then

$$
\bar{g}^{\prime}(x)-\bar{g}^{\prime}\left(x^{\prime}\right)<0 \text { and } \underline{g}^{\prime}(x)-\underline{g}^{\prime}\left(x^{\prime}\right)<0
$$

for all $x, x^{\prime} \in \mathbf{R}$ with $\left|x^{\prime}\right|<x$.

We study naturally our equation (2.1) by considering the Fourier coefficients of $u$ : For $p \in \mathbf{Z}^{d}$ let

$$
\hat{u}(p)=\int u(t) \exp (-2 \pi i p \cdot t) d t .
$$

The inverse transformation is given by Parseval's formula

$$
u(t)=\sum_{p \in \mathbf{Z}^{d}} \hat{u}(p) \exp (2 \pi i p \cdot t) .
$$

Here and in the sequel the equality is understood in the sense of $L_{2}\left(\mathbf{T}^{d}\right)$. Since $u$ and $w$ are real functions on $\mathbf{T}^{d}$, we have

$$
\hat{u}(-p)=\overline{\hat{u}(p)} \quad \text { and } \quad \hat{w}(-p)=\overline{\hat{w}(p)}
$$

in particular,

$$
\hat{u}(0) \in \mathbf{R} \text { and } \hat{w}(0) \in \mathbf{R} .
$$

By the convolution rule $\widehat{w * u}(p)=\hat{w}(p) \hat{u}(p),(2.1)$ can now be rewritten as

$$
u(t)=g\left\{\lambda \sum_{q \in \mathbf{Z}^{d}} \hat{w}(q) \hat{u}(q) \exp (2 \pi i q t)\right\}, \quad t \in \mathbf{T}^{d}
$$

or

$$
\hat{u}(p)=\left[g\left\{\lambda \sum_{q \in \mathbf{Z}^{d}} \hat{w}(q) \hat{u}(q) \exp (2 \pi i q t)\right\}\right]^{\wedge}(p)
$$

for all $p \in \mathbf{Z}^{d}$.

DEFINITION. We say that a solution $u$ of $(2.1)$ is $p$-stable, $p \in \mathbf{Z}^{d}$, if

$$
\lambda|\hat{w}(p)| \int \bar{g}^{\prime}\{\lambda w * u(t)\} d t<1 .
$$

It is called $p$-unstable or critical if

$$
\lambda|\hat{w}(p)| \int \underline{g}^{\prime}\{\lambda w * u(t)\} d t \geq 1
$$

We conclude this section with some simple results about solutions of (2.1):

(i) Set

$$
G(\lambda, u)=u-g\{\lambda w * u\}
$$

Then

$$
G(\lambda, 0)=0 \text { for all } \lambda \text {, }
$$


since (2.1) has always the trivial solution $u \equiv 0$. If $g^{\prime}(0)<+\infty$, the linearized operator at $u \equiv 0$ is given by

$$
D_{u} G(\lambda, 0) \cdot v=v-g^{\prime}(0) \lambda w * v
$$

with $v \in L_{2}\left(\mathbf{T}^{d}\right)$. The operator $v \rightarrow w * v$ is compact in $L_{2}\left(\mathbf{T}^{d}\right)$, so that the spectrum of $D_{u} G(\lambda, 0)$ is

$$
\operatorname{sp} D_{u} G(\lambda, 0)=\{1\} \cup \bigcup_{p \in \mathbf{Z}^{d}}\left\{1-g^{\prime}(0) \lambda \hat{w}(p)\right\} .
$$

Therefore by the implicit function theorem, there is no bifurcation for $\lambda$ not in

$$
\mathbf{R} \cap\left\{\left(g^{\prime}(0) \hat{w}(p)\right)^{-1}, p \in \mathbf{Z}^{d} \text { with } \hat{w}(p) \neq 0\right\} .
$$

(ii) There are two kinds of invariance for the set of solutions of (2.1): First, (2.1) is translation invariant; i.e., if $u$ is a solution of $(2.1)$, then so is

$$
u_{s}(t)=u(t+s)
$$

for all $s \in \mathbf{T}^{d}$, since

$$
G\left(\lambda, u_{s}\right)(t)=G(u, \lambda)(t+s)
$$

Recall that

$$
\widehat{u_{s}}(p)=\hat{u}(p) \exp \{2 \pi i p s\} \text {. }
$$

Second, if $u$ is a solution of $(2.1)$, so is $-u$, since

$$
G(\lambda,-u)=-G(\lambda, u) .
$$

THEOREM 1. (i) Let $g^{\prime}(0)<\infty$ and $\lambda \in\left(0,\left(g^{\prime}(0) \cdot\|w\|_{L^{1}}\right)^{-1}\right)$. Then (2.1) has only the trivial solution $u \equiv 0$.

(ii) Let $\hat{w}(0)>0$ and

$$
\lambda_{0}= \begin{cases}0 & \text { if } g^{\prime}(0)=+\infty \\ \left(g^{\prime}(0) \hat{w}(0)\right)^{-1} & \text { otherwise }\end{cases}
$$

At $\lambda_{0}$ a branch of constant nontrivial solutions $u_{\lambda} \equiv \pm \hat{u}_{\lambda}(0)$ bifurcates from the trivial solution, where $\hat{u}_{\lambda}(0)=\hat{u}(0)>0$ is the unique positive solution of

$$
\hat{u}(0)=g\{\lambda \hat{u}(0) \hat{w}(0)\}, \quad \lambda \in\left(\lambda_{0},+\infty\right) .
$$

If, moreover, $w \geq 0$, then this branch does not have secondary bifurcations.

PROOF. (i) $g$ being odd, we have for any solution $u$,

$$
\sup _{t}|u(t)|=\sup _{t} g\{\lambda|w * u(t)|\} \leq g\left\{\lambda\|w\|_{L^{1}} \sup _{t}|u(t)|\right\},
$$

which for $\lambda<\left(g^{\prime}(0)\|w\|_{L^{1}}\right)^{-1}$ implies $\sup _{t}|u(t)|=0$.

(ii) The first assertion of (ii) is well known (see, e.g., [2, Appendix B],). If $w \geq 0, w \not \equiv 0$, then $\hat{w}(p)<\hat{w}(0)$ for all $p \in \mathbf{Z}^{d}-\{0\}$. If $g$ is differentiable on $(0,+\infty)$, the spectrum of the linearization at $u_{\lambda} \equiv \hat{u}(0)$,

$$
D_{u} G(\lambda, \hat{u}(0)) v=v-\lambda g^{\prime}\{\lambda \hat{w}(0) \hat{u}(0)\} w * v
$$




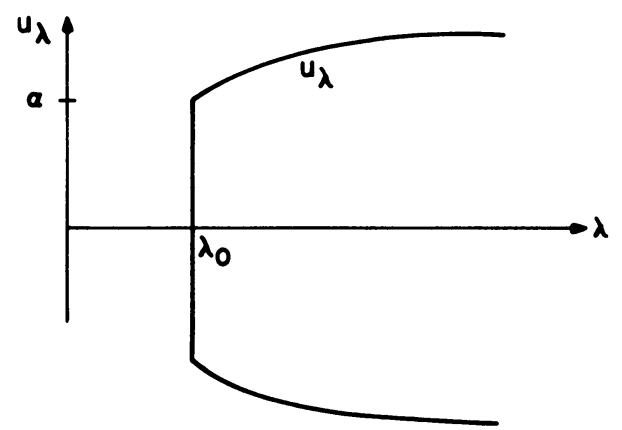

FIGURE 1

consists of the values 1 and $1-\lambda \hat{w}(p) g^{\prime}\{\lambda \hat{w}(0) \hat{u}(0)\}, p \in \mathbf{Z}^{d}$. But

$$
\lambda \hat{w}(p) g^{\prime}\{\lambda \hat{w}(0) \hat{u}(0)\}<\lambda \hat{w}(0) g^{\prime}\{\lambda \hat{w}(0) \hat{u}(0)\}=\frac{d}{d x} g\{\lambda x \hat{w}(0)\}_{\mid x=\hat{u}(0)}<1
$$

and the branch $u \equiv \pm \hat{u}(0)$ cannot have a secondary bifurcation. For a general function $g$, a simple approximation by a smooth function $\tilde{g}$ shows that there are no secondary bifurcations on $\pm u(0)$ for $g$ either.

REMARK. Let again $\hat{w}(0)>0$. We are interested in the behavior of $\hat{u}_{\lambda}(0)$ for $\lambda \searrow \lambda_{0}$. Assume first that $g$ is linear in some interval $[-\alpha,+\alpha], 0<\alpha<+\infty$; i.e.

$$
g(x)=g^{\prime}(0) x, \quad 0<g^{\prime}(0)<+\infty,
$$

for $x \in[-\alpha,+\alpha]$. Of course, we suppose $\alpha$ to be maximal with this property. Then at $\lambda=\lambda_{0}=\left(g^{\prime}(0) \hat{w}(0)\right)^{-1}$ we have in addition to (2.37) the constant solutions (see Figure 1)

$$
u_{\lambda_{0}} \equiv x \quad \text { with } x \in[-\alpha,+\alpha]
$$

Conversely, if $g$ is not linear in a neighborhood of 0 , then the concavity of $g$ implies that either

$$
\lambda_{0}=0 \text { and then } g\left\{\lambda_{0} \hat{w}(0) x\right\}=0 \text { for all } x,
$$

or

$$
\lambda_{0}>0 \text {, and then } 0<g^{\prime}(0)<+\infty
$$

and

$$
g\left\{\lambda_{0} \hat{w}(0) x\right\}<g^{\prime}(0) \lambda_{0} \hat{w}(0) x=x \quad \text { for all } x \in(0, \infty) .
$$

In both cases there are no nontrivial constant solutions at $\lambda_{0}$. This shows that we have, in addition to Theorem 1(ii), nontrivial constant solutions of (2.1) if and only if $g$ is linear in a neighborhood of 0 . (2.37) and (2.40) are the only nontrivial constant solutions of (2.1).

We set the maximal $\alpha$ from (2.39) equal to 0 if $g$ is not linear in a neighborhood of zero. It is then easy to see that

$$
\lim _{\lambda \searrow \lambda_{0}} \hat{u}_{\lambda}(0)=\lim _{x \searrow \alpha} g(x)
$$

In particular, if $g$ is continuous at 0 , but not linear in a neighborhood of 0 , then

$$
\lim _{\lambda \searrow \lambda_{0}} \hat{u}_{\lambda}(0)=0
$$


and graphically the nontrivial constant solutions branch indeed from the trivial solution.

Finally, we note some simple properties of the function $\lambda \rightarrow \hat{u}_{\lambda}(0)$ if $\hat{w}(0)>0$. For $\lambda \in\left(\lambda_{0},+\infty\right)$ we set

$$
\phi_{0}(\lambda, x)=g\{\lambda \hat{w}(0) x\}
$$

and

$$
\bar{\partial} \phi_{0}(\lambda, x)=\lambda \hat{w}(0) \bar{g}^{\prime}\{\lambda \hat{w}(0) x\} .
$$

On $\left(\lambda_{0},+\infty\right)$ the function $\lambda \rightarrow \hat{u}_{\lambda}(0)=\phi_{0}\left(\lambda, \hat{u}_{\lambda}(0)\right)$ is continuous and increasing with

$$
\lim _{\lambda \rightarrow+\infty} \hat{u}_{\lambda}(0)=\gamma
$$

where $\gamma$ stems from (2.14). The solution $u_{\lambda} \equiv \pm \hat{u}_{\lambda}(0)$ is 0 -stable since

$$
0 \leq \bar{\partial} \phi_{0}\left(\lambda, \hat{u}_{\lambda}(0)\right)<1 \text {. }
$$

Moreover,

$$
\lim _{\lambda \rightarrow+\infty} \bar{\partial} \phi_{0}\left(\lambda, \hat{u}_{\lambda}(0)\right)=0 .
$$

To see the last equality, we fix $0<\hat{x}<\underline{\gamma}$ and $\bar{\lambda}>\lambda_{0}$ with $u_{0}(\bar{\lambda})>\bar{x}$ by (2.46). The concavity of $g$ on $\mathbf{R}^{+}$shows for $\lambda>\bar{\lambda}$ that

$$
0 \leq \bar{\partial} \phi_{0}\left(\lambda, \hat{u}_{\lambda}(0)\right) \leq\left(g\left\{\lambda \hat{w}(0) \hat{u}_{\lambda}(0)\right\}-g\{\lambda \hat{w}(0) \bar{x}\}\right) /\left(\hat{u}_{\lambda}(0)-\bar{x}\right) .
$$

By (2.14) the right side of (2.47) goes to zero as $\lambda \rightarrow+\infty$.

3. Some invariance results for the Fourier coefficients. In this section, we show that the conditions on $g$ imply the existence of classes of functions $u$, characterized by their Fourier coefficients, which are invariant under the operation $u \rightarrow g\{\lambda w * u\}$. Therefore, solutions of (2.1) can be studied independently in each of these classes.

Let us define, for $p \in \mathbf{Z}^{d}$ and $A \subseteq \mathbf{Z}$,

$$
A p=\{k p, k \in A\} .
$$

Proposition 1. Let $p, q \in \mathbf{Z}^{d}$ and $m$ be an integrable function on $\mathbf{T}^{d}$. Then

$$
\hat{m}(r)=0 \quad \text { for all } r \notin \mathbf{Z} p
$$

if and only if

$$
m(\cdot+s)=m(\cdot) \quad \text { for all } s \in \mathbf{T}^{d} \text { with } p \cdot s \equiv 0 \bmod 1
$$

$$
\hat{m}(r)=0 \quad \text { for all } r \notin(2 \mathbf{Z}+1) p
$$

if and only if

$$
m(\cdot+s)=-m(\cdot) \quad \text { for all } s \in \mathbf{T}^{d} \text { with } p \cdot s \equiv \frac{1}{2} \bmod 1 .
$$

(iii) Assume that $p \neq 0$ and $q \neq 0$ are not collinear; i.e.,

$$
(p p)(q q)-(p q)^{2}>0 \text {. }
$$


Then

$$
\hat{m}(r)=0 \quad \text { for all } r \notin[(2 \mathbf{Z}+1) p+2 \mathbf{Z} q] \cup[2 \mathbf{Z} p+(2 \mathbf{Z}+1) q]
$$

if and only if

$$
m(\cdot+s)=m(\cdot) \quad \text { for all } s \in \mathbf{T}^{d} \text { with } p s \bmod 1 \equiv q s \bmod 1 \equiv \frac{1}{2} .
$$

(iv) Assume that $p, q \in \mathbf{Z}^{d} \backslash\{0\}$ are collinear; i.e.,

$$
n_{1} p=n_{2} q \neq 0
$$

for some $n_{1}, n_{2} \in \mathbf{Z} \backslash\{0\}$ with $\operatorname{gcd}\left(n_{1}, n_{2}\right)=1$. Here, gcd denotes the greatest common divisor. Set $r_{0}=p / n_{2}=q / n_{1}$. Then $r_{0} \in \mathbf{Z}^{d}$, and

$$
[(2 \mathbf{Z}+1) p+2 \mathbf{Z} q] \cup[2 \mathbf{Z} p+(2 \mathbf{Z}+1) q]= \begin{cases}\mathbf{Z} r_{0} & \text { if } n_{1} \cdot n_{2} \text { even }, \\ (2 \mathbf{Z}+1) r_{0} & \text { if } n_{1} \cdot n_{2} \text { odd. }\end{cases}
$$

Now, if $n_{1} \cdot n_{2}$ is odd, then

(3.7) is equivalent to (3.8).

But if $n_{1} \cdot n_{2}$ is even, then (3.7) is equivalent to

$$
m(\cdot+s)=m(\cdot) \quad \text { for all } s \in \mathbf{T}^{d} \text { with } p s \bmod 1 \equiv q s \bmod 1 \equiv 0 .
$$

ProOF. (i) (3.2) and (2.20) imply (3.3) immediately. Conversely, by (3.3) and (2.34), we get for all $s \in \mathbf{T}^{d}$ with $p s \equiv 0 \bmod 1$ and $r \in \mathbf{Z}^{d}$ that

$$
\hat{m}(r)[\exp \{2 \pi i r s\}-1]=0 \text {. }
$$

Let $r=\left(r_{1}, \ldots, r_{d}\right)$ with $\hat{m}(r) \neq 0$. Then

$$
r \cdot s \equiv 0 \bmod 1 \quad \text { for all } s \in \mathbf{T}^{d} \text { with } p s \equiv 0 \bmod 1 \text {. }
$$

Considering, in particular, $s=\left(0, \ldots, s_{k}, 0, \ldots\right)$, we have $r_{k} s_{k} \in \mathbf{Z}$ for all $s_{k}$ with $p_{k} s_{k} \in \mathbf{Z}$, which can only hold if $r_{k}=n_{k} p_{k}$ for some $n_{k} \in \mathbf{Z}$. Moreover, if $n_{k} \neq n_{l}$ for $k \neq l$ and $p_{k}, p_{l} \neq 0$, we take $s=\left(0, \ldots, s_{k}, \ldots, s_{l}, 0, \ldots\right)$ with $s_{k}=\frac{1}{2}\left(n_{k}-n_{l}\right) p_{k}$ and $s_{l}=-\frac{1}{2}\left(n_{k}-n_{l}\right) p_{l}$, which satisfies $p s=0$ but $r s=\frac{1}{2}$. We have a contradiction to (3.13). Therefore, $n_{k}=n_{l}$ and $r \in \mathbf{Z} p$.

(ii) Obviously, if (3.4) is satisfied, then so is (3.5).

Let (3.5) hold. Then (3.3) holds also, and we get $\hat{m}(r)=0$ for all $r \notin \mathbf{Z} p$. But if $r=2 n p \neq 0$ with $n \in \mathbf{Z}$, we take $s=\left(0, \ldots, 1 / 2 p_{k}, 0, \ldots\right)$ for some $k$ with $p_{k} \neq 0$, such that $p s=\frac{1}{2}$. By (3.5)

$$
0=\hat{m}(r)[\exp \{2 \pi i r s\}+1]=\hat{m}(r) 2,
$$

which shows $\hat{m}(r)=0$. Thus (3.4) holds.

(iii) Evidently, (3.8) follows from (3.7). Conversely, assume (3.8). First, one checks that the set of all $s$ satisfying $p s \equiv q s \equiv \frac{1}{2} \bmod 1$ is given by

$$
\begin{aligned}
s= & \frac{1}{2}\left((p p)(q q)-(p q)^{2}\right)^{-1} \\
& \times\{p[(2 k+1)(q q)-(2 l+1)(p q)]+q[(2 l+1)(p p)-(2 k+1)(p q)]\}+\tilde{s}
\end{aligned}
$$

with $k, l \in \mathbf{Z}$ and $(\tilde{s} p)=(\tilde{s} q)=0$. (3.8) says that if $\hat{m}(r) \neq 0$ then $r \cdot s \equiv \frac{1}{2} \bmod 1$ for all $s$ from (3.14). Setting $r=\alpha p+\beta q+\tilde{r}$ with $\tilde{r} p=\tilde{r} q=0$, we find for all such $s$ that

$$
r s=\frac{1}{2}[\alpha(2 k+1)+\beta(2 l+1)]+\tilde{r} \tilde{s} \equiv \frac{1}{2} \bmod 1
$$


for all $k, l \in \mathbf{Z}$ and all $\tilde{s}$. Hence, $\tilde{r}=0$ and

$$
(\alpha, \beta) \in[(2 \mathbf{Z}+1) \times 2 \mathbf{Z}] \cup[2 \mathbf{Z} \times(2 \mathbf{Z}+1)],
$$

which is $r \in[(2 \mathbf{Z}+1) p+2 \mathbf{Z} q] \cup[2 \mathbf{Z} p+(2 \mathbf{Z}+1) q]$.

(iv) Since $\operatorname{gcd}\left(n_{1}, n_{2}\right)=1$, there exists $k_{1}, k_{2} \in \mathbf{Z}$ with

$$
k_{1} n_{1}+k_{2} n_{2}=1 \text {. }
$$

Therefore

$$
k_{1} q+k_{2} p=r_{0} \in \mathbf{Z}^{d} .
$$

Now if $n_{1}$ and $n_{2}$ are odd, then

$$
l_{1} n_{1}+l_{2} n_{2} \in(2 \mathbf{Z}+1) \quad \text { iff } \quad\left(l_{1}, l_{2}\right) \in[(2 \mathbf{Z}+1) \times 2 \mathbf{Z}] \cup[2 \mathbf{Z} \times(2 \mathbf{Z}+1)],
$$

which by $l_{1} q+l_{2} p=\left(l_{1} n_{1}+l_{2} n_{2}\right) r_{0}$ shows (3.10) for $n_{1} \cdot n_{2}$ odd. On the other hand, if $n_{1} \cdot n_{2}$ is even-i.e., $\left(n_{1}, n_{2}\right) \in[(2 \mathbf{Z}+1) \times 2 \mathbf{Z}] \cup[2 \mathbf{Z} \times(2 \mathbf{Z}+1)]$-then

$$
l_{1} q+l_{2} p=\left(l_{1}+n_{2}\right) q+\left(l_{2}-n_{1}\right) p=\left(l_{1} n_{1}+l_{2} n_{2}\right) r_{0}
$$

shows

$$
[(2 \mathbf{Z}+1) p+2 \mathbf{Z} q] \cup[2 \mathbf{Z} p+(2 \mathbf{Z}+1) q]=\mathbf{Z} p+\mathbf{Z} q=\mathbf{Z} r_{0}
$$

Now, let $n_{1} \cdot n_{2}$ be odd. Then, since $\left(k_{1}, k_{2}\right) \in[(2 \mathbf{Z}+1) \times 2 \mathbf{Z}] \cup[2 \mathbf{Z} \times(2 \mathbf{Z}+1)]$ by $(3.18)$,

$$
p s \equiv q s \equiv \frac{1}{2} \bmod 1 \quad \text { iff } \quad r_{0} s \equiv \frac{1}{2} \bmod 1,
$$

and (ii) shows the equivalence of (3.7) and (3.8). It is clear that

$$
p s \equiv q s \equiv 0 \bmod 1 \quad \text { iff } \quad r_{0} s \equiv 0 \bmod 1 .
$$

If $n_{1} \cdot n_{2}$ is even, then (3.19) and (i) show that (3.7) and (3.11) are equivalent.

DEFINITION. We denote by $\mathcal{F}_{p}, \mathcal{F}_{p}^{\prime}$ and $\mathcal{F}_{p q}$ the sets of integrable functions on $\mathbf{T}^{d}$ which satisfy (3.2), (3.4), and (3.7) respectively.

We note some immediate consequences of the proposition:

COROLlaRY. If $w \in \mathcal{F}_{p}, w \in \mathcal{F}_{p}^{\prime}$, or $w \in \mathcal{F}_{p q}$ for noncollinear $p$ and $q$, then all solutions of (2.1) are in $\mathcal{F}_{p}, \mathcal{F}_{p}^{\prime}$, and $\mathcal{F}_{p q}$, respectively.

REMARK. For $p \in \mathbf{Z}^{d} \backslash\{0\}$ set

$$
w_{p}(t)=\sum_{r \notin(2 \mathbf{Z}+1) p \backslash\{ \pm p\}} \hat{w}(r) \exp \{2 \pi i r t\} .
$$

If $w=w_{p}$, i.e., if

$$
\hat{w}(r)=0 \quad \text { for all } r \in(2 \mathbf{Z}+1) p \backslash\{+p,-p\},
$$

then any function $u \in \mathcal{F}_{p}^{\prime}$ is a solution of (2.1) if and only if

$$
u(t)=g\left\{\lambda \sum_{r= \pm p} \hat{w}(r) \hat{u}(r) \exp (2 \pi i r t)\right\} .
$$

Of course, the last statement also holds trivially for $p=0 . \mathcal{F}_{0}=\mathcal{F}_{0}^{\prime}$ consists only of constant functions, and $u \equiv \hat{u}(0)$ is a solution of $(2.1)$ if and only if $\hat{u}(0)$ satisfies

$$
\hat{u}(0)=g\{\lambda \hat{w}(0) \hat{u}(0)\} \text {. }
$$


Similarly, for $p, q \in \mathbf{Z}^{d}, p \neq q \neq 0$, we set

$$
\begin{aligned}
w_{p q}= & \sum_{r= \pm p, \pm q} \hat{w}(r) \exp \{2 \pi i r t\} \\
& +\sum_{r \notin[(2 \mathbf{Z}+1) p+2 \mathbf{Z} q] \cup[2 \mathbf{Z} p+(2 \mathbf{Z}+1) q]} \hat{w}(r) \exp \{2 \pi i r t\} .
\end{aligned}
$$

If $w=w_{p q}$, i.e., if

(3.25) $\hat{w}(r)=0$ for all $r \in([(2 \mathbf{Z}+1) p+2 \mathbf{Z} q] \cup[2 \mathbf{Z} p+(2 \mathbf{Z}+1) q]) \backslash\{ \pm p, \pm q\}$,

then any function $u \in \mathcal{F}_{p q}$ is a solution of $(2.1)$ if and only if

$$
u(t)=g\left\{\lambda \sum_{r= \pm p, \pm q} \hat{w}(r) \hat{u}(r) \exp (2 \pi i r t)\right\} .
$$

The simple forms of (3.22), (3.23), and (3.26) lead to the following definitions.

DEFINITION. A function $u \in \mathcal{F}_{p}^{\prime}$ is called a $p$-primary solution, $p \in \mathbf{Z}^{d}$, if

$$
\hat{u}(p) \neq 0
$$

and

$$
u(t)=g\left\{\lambda w_{p} * u(t)\right\}
$$

with $w_{p}$ from (3.20). $u \in \mathcal{F}_{p q}$ is called a $(p, q)$-secondary solution if

$$
\hat{u}(p) \neq 0, \quad \hat{u}(q) \neq 0,
$$

and

$$
u(t)=g\left\{\lambda w_{p q} * u(t)\right\}
$$

with $w_{p q}$ from (3.24).

Note in particular that $p$-primary solutions and $(p, q)$-secondary solutions are, in general, not solutions of (2.1) unless $w=w_{p}, w=w_{p q}$, respectively. $p$-primary solutions and $(p, q)$-secondary solutions are nontrivial by definition. Only for $p=0$, the 0 -primary solutions are always the nontrivial constant solutions of $(2.1)$, which are treated in Theorem 1.

For $p \neq 0$ we investigate $p$-primary solutions in the next section. $(p, q)$-secondary solutions are studied in $\S \S 5$ and 7.

4. Primary solutions. For $p \in \mathbf{Z}^{d} \backslash\{0\}$ we shall study the existence of (nontrivial) $p$-primary solutions in $\xi_{p}^{\prime}$, i.e., solutions of (3.28). This means implicitly that we assume $w=w_{p}$ with $w_{p}$ from (3.20) or that (3.21) holds. Let us define (assuming for a moment that $g$ is a function on $\mathbf{C}$ )

$$
\begin{aligned}
\Phi_{p}\left(\lambda, z_{p}, z_{-p}\right) & =\int g\left\{\lambda \sum_{q= \pm p} \hat{w}(q) z_{q} \exp \{2 \pi i q t\}\right\} \exp \{-2 \pi i p t\} d t \\
\phi_{p}(\lambda, z) & =\operatorname{Re} \Phi_{p}(\lambda, z, \bar{z}) \\
& =\int g\{\lambda 2 \operatorname{Re}(\hat{w}(p) z \exp (2 \pi i p t))\} \cos (2 \pi p t) d t
\end{aligned}
$$


and its 'formal' symmetric derivative

$$
\begin{aligned}
\bar{\partial} \phi_{p}(\lambda, z) & =\frac{1}{2}\left(\bar{\partial}_{z_{p}} \Phi_{p}+\bar{\partial}_{z_{-p}} \Phi_{-p}\right)(\lambda, z, \bar{z}) \\
& =\lambda \operatorname{Re} \hat{w}(p) \int \bar{g}^{\prime}\{\lambda 2 \operatorname{Re}(\hat{w}(p) z \exp (2 \pi i p t))\} d t
\end{aligned}
$$

Note that even if $\hat{w}(p)=\hat{w}(-p) \in \mathbf{R}$ and $\bar{z}=z \in \mathbf{R}$,

$$
\begin{aligned}
\bar{\partial} \phi_{p}(\lambda, z) & \neq \frac{\bar{\partial}}{\partial z} \phi_{p}(\lambda, z) \\
& =\lambda \hat{w}(p) \int \bar{g}^{\prime}\{\lambda \hat{w}(p) z 2 \cos (2 \pi p t)\} 2 \cos ^{2}(2 \pi p t) d t
\end{aligned}
$$

The following result is generalized in $\S 5$.

THEOREM 2. If $\operatorname{Im} \hat{w}(p) \neq 0$, then there do not exist p-primary solutions in $\mathcal{F}_{p}^{\prime}$.

REMARK. We know already from (2.31) that the condition of Theorem 2 is necessary for local bifurcations from zero. The theorem and its generalization in $\S 5$ is more interesting as a gobal result. It has nothing to do with our restriction to $\lambda \in \mathbf{R}_{+}$: If $\operatorname{Im} \hat{w}(p) \neq 0$, then there are no $p$-primary solutions even for all $\lambda \in \mathbf{R}$. However, the restriction to $\lambda \in \mathbf{R}_{+}$makes it necessary to have $\hat{w}(p)>0$, since we must have $\lambda \hat{w}(p)>0$ for the existence of $p$-primary solutions, as can be seen from (3.22).

In this context we want to mention that in [2] the assumptions of Theorems 1.2 and 2.3 have been formulated somewhat sloppily. Instead of simply supposing $\nu \neq 0$, we must demand $\nu>0$, as in the proof there (see also [2, p. 336]). Thus, we get the following assumptions for the existence of $p$-primary solutions in $\mathcal{F}_{p}^{\prime}$ with $\lambda \in \mathbf{R}_{+}$.

THEOREM 3. For $p \in \mathbf{Z}^{d} \backslash\{0\}$ let

$$
0<\hat{w}(p) \in \mathbf{R} \text { and } \hat{w}(r)=0 \text { for all } r \in(2 \mathbf{Z}+1) p \backslash\{+p,-p\} .
$$

Define

$$
\lambda_{p}= \begin{cases}0 & \text { if } g^{\prime}(0)=+\infty, \\ \left(g^{\prime}(0) \hat{w}(p)\right)^{-1} & \text { if } 0<g^{\prime}(0)<+\infty\end{cases}
$$

With exceptions for $\lambda=\lambda_{p}$, the functions

$$
u_{p}^{s}(\lambda, t)=g\left\{\lambda \hat{w}(p)\left|\hat{u}_{\lambda}(p)\right| 2 \cos (2 \pi p(t+s))\right\},
$$

$\lambda \in\left(\lambda_{p},+\infty\right), s \in \mathbf{T}^{d}$, are the only p-primary solutions of $(2.1)$, where $|\hat{u}(p)|=$ $\left|\hat{u}_{\lambda}(p)\right|>0$ is the unique positive solution of

$$
|\hat{u}(p)|=\int g\{\lambda \hat{w}(p)|\hat{u}(p)| 2 \cos (2 \pi p t)\} \cos (2 \pi p t) d t .
$$

$\lambda \rightarrow|\hat{u}(p)|$ is continuous and increasing on $\left(\lambda_{p},+\infty\right)$ with

$$
\lim _{\lambda \rightarrow \infty}|\hat{u}(p)|=\frac{2 \gamma}{\pi} .
$$

The p-primary solutions (4.7) are p-stable; we even have

$$
\bar{\partial} \phi_{p}(\lambda,|\hat{u}(p)|) \in\left(\frac{1}{2}, 1\right), \quad \lambda \in\left(\lambda_{p},+\infty\right),
$$


and

$$
\lim _{\lambda \rightarrow \infty} \bar{\partial} \phi_{p}(\lambda,|\hat{u}(p)|)=\frac{1}{2} .
$$

REMARK. At $\lambda=\lambda_{p}$ there are (nontrivial) $p$-primary solutions if and only if $g$ is linear on some interval $[-\alpha,+\alpha]$, with $\alpha$ maximal (see (2.39)). If the latter is the case, all $p$-primary solutions at $\lambda=\lambda_{p}$ are of the form

$$
u_{p}^{s}\left(\lambda_{p}, t\right)=y 2 \cos (2 \pi p(t+s))
$$

with $y \in(0, \alpha / 2], s \in \mathbf{T}^{d}$.

PROOF. For $z>0$ the function $\phi_{p}(\lambda, \cdot)$ is positive and concave. Excluding the case $\lambda=\lambda_{p}$, treated in the remark, there exists a unique positive fixed point $|\hat{u}(p)|$ of $\phi_{p}(\lambda, \cdot)$ if and only if $\lambda g^{\prime}(0) \hat{w}(p)>1$, or, equivalently, if $\lambda>\lambda_{p} \cdot|\hat{u}(p)|$ is increasing in $\lambda$.

(4.9) is evident. Obviously, (4.7) is a $p$-primary solution. Conversely, if $v$ is any $p$-primary solution at $\lambda$ with $\hat{v}(p) \neq 0$, then $|\hat{v}(p)|$ is a positive fixed point of $\phi_{p}(\lambda, \cdot)$. Excluding $\lambda=\lambda_{p}$, we must have $\lambda>\lambda_{p}$ and $|\hat{v}(p)|=|\hat{u}(p)|$ such that $v$ has the form (4.7). To prove (4.10), we first show

$$
\lambda \hat{w}(p) \int \bar{g}^{\prime}\{\lambda \hat{w}(p)|\hat{u}(p)| 2 \cos (2 \pi p t)\} \sin ^{2}(2 \pi p t) d t=\frac{1}{2} .
$$

For this purpose we approximate $g$ uniformly by a differentiable function $\tilde{g}$ with the same properties as $g$. Assuming without loss of generality that $p_{1} \neq 0$, we get by partial integration that

$$
\begin{aligned}
\lambda \hat{w}(p) & \int_{\mathbf{T}^{d}} \tilde{g}^{\prime}\{\lambda \hat{w}(p)|\hat{u}(p)| 2 \cos (2 \pi p t)\} \sin ^{2}(2 \pi p t) d t \\
= & -\int_{\mathbf{T}^{d-1}}\left[\tilde{g}\{\cdots\} \sin (2 \pi p t) / 2|\hat{u}(p)| 2 \pi p_{1}\right]_{t_{1}=0}^{t_{1}=1} d\left(t_{2}, \ldots, t_{d}\right) \\
& +\frac{1}{2|\hat{u}(p)|} \int_{\mathbf{T}^{d}} \tilde{g}\{\cdots\} \cos (2 \pi p t) d t .
\end{aligned}
$$

The argument of the $\{\cdots\}$ is always the same as in the first line. Since the second term in (4.14) vanishes and the last term tends to $\frac{1}{2}$ as $\tilde{g}$ tends to $g,(4.13)$ is proved. Now the concavity of $\phi_{p}(\lambda, z)$ for $z>0$ yields, with (4.4),

$$
\bar{\partial} \phi_{p}(\lambda,|\hat{u}(p)|)=\frac{1}{2}\left(1+\frac{\bar{\partial}}{\partial z} \phi_{p}(\lambda,|\hat{u}(p)|)\right) \in\left(\frac{1}{2}, 1\right),
$$

which is (4.10). For (4.11) we have to show by the last equality that

$$
\lim _{\lambda \rightarrow \infty} \frac{\bar{\partial}}{\partial z} \phi_{p}(\lambda,|\hat{u}(p)|)=0
$$

Fix $z_{0} \in(0,2 \gamma / \pi)$ such that, by $(4.9),|\hat{u}(p)|>z_{0}$ for all sufficiently large $\lambda$. The concavity of $\phi_{p}(\lambda, z)$ for $z>0$ implies

$$
\begin{aligned}
0 & \leq \frac{\bar{\partial}}{\partial z} \phi_{p}(\lambda,|\hat{u}(p)|) \\
& \leq \int\left[g\{\lambda \hat{w}(p)|\hat{u}(p)| 2 \cos (2 \pi p t)\}-g\left\{\lambda \hat{w}(p) z_{0} 2 \cos (2 \pi p t)\right\}\right] \\
& \times \cos (2 \pi p t) d t /\left(|\hat{u}(p)|-z_{0}\right) \rightarrow 0 \text { for } \lambda \rightarrow \infty .
\end{aligned}
$$

This shows (4.16), and the proof is complete. 
5. Secondary bifurcations for $p, q$ noncollinear. For the rest of the paper we assume that

$$
0<g^{\prime}(0)<+\infty
$$

and

$$
g \text { is strictly concave on }(0,+\infty) \text {. }
$$

This does not allow $g$ to be linear or constant on some interval. In particular, $g$ is strictly increasing. For convenience we suppose, moreover, that $g$ is differentiable on $\mathbf{R}$, though this condition is not really necessary and can be overcome by approximating $g$ suitably (see the proof of Theorem 4 for such an approximation). We study the following problem: In which cases do there exist secondary bifurcating branches of solutions from branches of primary solutions? We restrict this problem to the investigation of $(p, q)$-secondary solutions. Moreover, we assume in this section that $p, q \in \mathbf{Z}^{d}$ are noncollinear in the sense that

$$
\text { either } p=0 \neq q \text { or }(p p)(q q)-(p q)^{2}>0 \text {. }
$$

Secondary bifurcations for collinear $p, q \neq 0$ are studied in $\S 7$.

In the following theorems the formulas for $(0, q)$-secondary solutions, $q \neq 0$, and for $(p, q)$-secondary solutions, $p, q \in \mathbf{Z}^{d} \backslash\{0\}$ noncollinear, are different. The proofs, however, follow the same lines. If necessary, we use square brackets containing two lines, the first of which corresponds to $p=0$ and the second to $p \neq 0$; for example,

$$
\sum_{r= \pm p} \hat{w}(r) \exp \{2 \pi i r t\}=\left[\begin{array}{l}
\hat{w}(0) \\
2 \operatorname{Re}(\hat{w}(p) \exp \{2 \pi i p t\})
\end{array}\right] \text {. }
$$

We prove the following generalization of Theorem 2 .

THEOREM 4. Let $p, q \in \mathbf{Z}^{d}$ satisfy (5.3), let (3.25) hold and $\hat{w}(p) \neq 0, \hat{w}(q) \neq 0$. There exist $(p, q)$-secondary solutions only if

$$
\operatorname{Im} \hat{w}(p)=0, \quad \operatorname{Im} \hat{w}(q)=0, \quad \text { and } \quad \hat{w}(p)>0, \hat{w}(q)>0 .
$$

ProOF. Let $v$ be a $(p, q)$-secondary solution. By assumption (5.3) we can find $s \in \mathbf{T}^{d}$ with

$$
\left[\begin{array}{l}
s \text { arbitrary } \\
2 \pi s p=-\arg (\hat{w}(p) \hat{v}(p))
\end{array}\right], \quad 2 \pi s q=-\arg (\hat{w}(q) \hat{v}(q)) .
$$

After a rotation of $u$ by $s$, we have

$$
v^{s}(t)=g\left\{\lambda\left[\begin{array}{l}
\hat{w}(0) \hat{v}(0) \\
|\hat{w}(p) \hat{v}(p)| 2 \cos (2 \pi p t)
\end{array}\right]+\lambda|\hat{w}(q) \hat{v}(q)| 2 \cos (2 \pi q t)\right\},
$$

such that $v^{s}$ is even and therefore $\widehat{v^{s}}(p) \in \mathbf{R} \backslash\{0\}$ and $\widehat{v^{s}}(q) \in \mathbf{R} \backslash\{0\}$. Since $v^{s}$ is a $(p, q)$-secondary solution, too, and $g$ is invertible as a strictly increasing function, we find for all $t \in \mathbf{T}^{d}$ that

$$
\begin{aligned}
& {\left[\begin{array}{l}
\hat{w}(0) \hat{v}^{s}(0) \\
\hat{v}^{s}(p) 2 \operatorname{Re}(\hat{w}(p) \exp (2 \pi i p t))
\end{array}\right]+\hat{v}^{s}(q) 2 \operatorname{Re}(\hat{w}(q) \exp (2 \pi i q t))} \\
& \quad=\left[\begin{array}{l}
\hat{w}(0) \hat{v}(0) \\
|\hat{w}(p) \hat{v}(p)| 2 \cos (2 \pi p t)
\end{array}\right]+|\hat{w}(q) \hat{v}(q)| 2 \cos (2 \pi q t),
\end{aligned}
$$

and therefore $\hat{w}(p) \in \mathbf{R}, \hat{w}(q) \in \mathbf{R}$. 
We know by Proposition 1(ii) that

$$
\left[g\left\{\lambda \hat{w}(p) \hat{v}^{s}(p)\left[\begin{array}{l}
1 \\
2 \cos (2 \pi p t)
\end{array}\right]\right\}\right]^{\wedge}(q)=0
$$

and

$$
\left[g\left\{\lambda \hat{w}(q) \hat{v}^{s}(q) 2 \cos (2 \pi q t)\right\}\right]^{\wedge}(p)=0 .
$$

Therefore

$$
\begin{aligned}
& 0 \neq \hat{v}^{s}(p) \\
& =\int\left(g \left\{\lambda \hat{w}(p) \hat{v}^{s}(p)\left[\begin{array}{l}
1 \\
2 \cos (2 \pi p t)
\end{array}\right]\right.\right. \\
& \left.+\lambda \hat{w}(q) \hat{v}^{s}(q) 2 \cos (2 \pi q t)\right\} \\
& \left.-g\left\{\lambda \hat{w}(q) \hat{v}^{s}(q) 2 \cos (2 \pi q t)\right\}\right) \cos (2 \pi p t) d t \\
& 0 \neq \hat{v}^{s}(q) \\
& =\int\left(g \left\{\lambda \hat{w}(p) \hat{v}^{s}(p)\left[\begin{array}{l}
1 \\
2 \cos (2 \pi p t)
\end{array}\right]\right.\right. \\
& \left.+\lambda \hat{w}(q) \hat{v}^{s}(q) 2 \cos (2 \pi q t)\right\} \\
& \left.-g\left\{\lambda \hat{w}(p) \hat{v}^{s}(p)\left[\begin{array}{l}
1 \\
2 \cos (2 \pi p t)
\end{array}\right]\right\}\right) \cos (2 \pi q t) d t .
\end{aligned}
$$

But both equations can only hold if $\lambda \hat{w}(p)>0$ and $\lambda \hat{w}(q)>0$. By our restriction to $\lambda>0$ we find the second part of assertion (5.5).

If we now assume (5.5) in addition to (3.18), we know by Theorems 1 and 3 that on $\left(\max \left(\lambda_{p}, \lambda_{q}\right),+\infty\right)$ we have both $p$-primary and $q$-primary solutions. On secondary bifurcations we get the following result, which will be proved at the end of $\S 8$. By $|\hat{u}(p)|=\left|\hat{u}_{\lambda}(p)\right|$ we denote the unique positive solution of (4.8), and (2.37), respectively, on $\left(\lambda_{p},+\infty\right)$.

THEOREM 5. Let $p, q \in \mathbf{Z}^{d}$ satisfy (5.3), and let (3.25) hold with $\hat{w}(p)>$ $0, \hat{w}(q)>0$.

(i) If $\frac{1}{2}>\hat{w}(q) / \hat{w}(p)>0$, then in $\mathcal{F}_{p q}$ there is no secondary bifurcation on the branch of p-primary solutions or on the branch of q-primary solutions.

(ii) If $1>\hat{w}(q) / \hat{w}(p)>\frac{1}{2}$, then in $\mathcal{F}_{p q}$ there is no secondary bifurcation on the p-primary branch, but on the q-primary branch there occurs a secondary bifurcation of a branch of $(p, q)$-secondary solutions at

$$
\lambda_{q p}=\inf \left\{\lambda>\lambda_{q} ; \partial \phi_{q}\left(\lambda^{\prime}\left|\hat{u}_{\lambda^{\prime}}(q)\right|\right)<\hat{w}(q) / \hat{w}(p) \text { for all } \lambda^{\prime}>\lambda\right\},
$$

with

$$
0<\lambda_{p}<\lambda_{q}<\lambda_{q p}<+\infty
$$




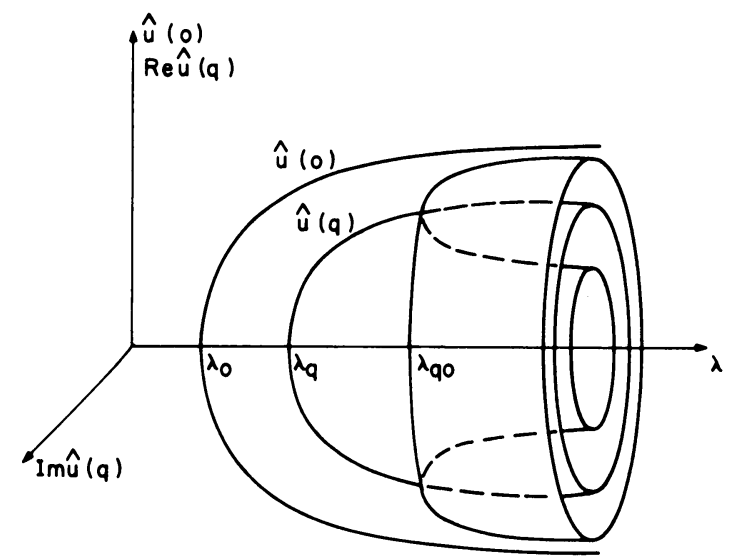

FIGURE 2. Bifurcations for $2 \hat{w}(q)>\hat{w}(0)>\hat{w}(q)>0$. The numerically exact picture is given in Example 3.3 of [24]

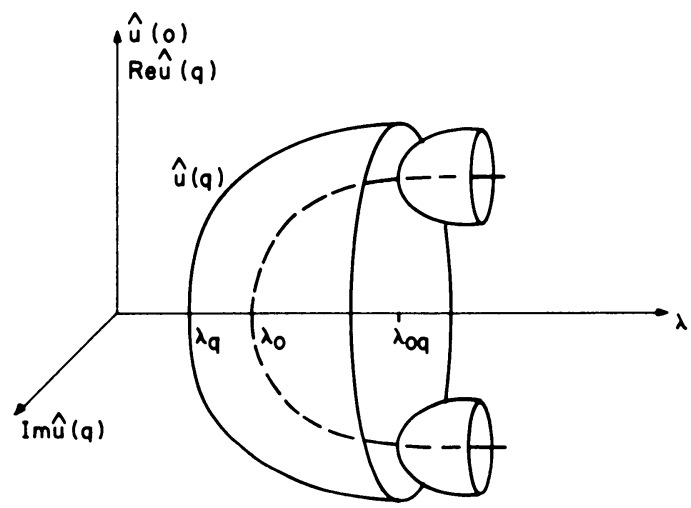

FIGURE 3. Bifurcations for $\hat{w}(q)>\hat{w}(0)>0$

This branch exists for all $\lambda \in\left(\lambda_{q p},+\infty\right)$ and consists of $(p, q)$-secondary solutions of the form

$$
\begin{array}{r}
u(t)=g\left\{\lambda \hat{w}(p)|\hat{v}(p)|\left[\begin{array}{l} 
\pm 1 \\
2 \cos (2 \pi p(t+s))
\end{array}\right]\right. \\
\quad+\hat{w}(q)|\hat{v}(q)| 2 \cos (2 \pi q(t+s))\}
\end{array}
$$

with $s \in \mathbf{T}^{d}$ (recall $|\hat{v}(p)| \neq 0$ and $|\hat{v}(q)| \neq 0$, by definition).

(iii) If $p=0$ and $1>\hat{w}(0) / \hat{w}(q)>0$, then in $\mathcal{F}_{0 q}$ there exists no secondary bifurcation on the branch of q-primary solutions, but on the branch of nontrivial constant solutions there occurs a secondary bifurcation of a branch of $(0, q)$-secondary solutions at

$$
\lambda_{0 q}=\inf \left\{\lambda, \partial \phi_{0}\left(\lambda^{\prime}, \hat{u}_{\lambda^{\prime}}(0)\right)<\hat{w}(0) / \hat{w}(q) \text { for all } \lambda^{\prime}>\lambda\right\}
$$


with

$$
0<\lambda_{q}<\lambda_{0}<\lambda_{0 q}<+\infty .
$$

This branch exists for all $\lambda \in\left(\lambda_{0 q},+\infty\right)$ and consists of $(0, q)$-secondary solutions of the form (5.12) with $p=0$.

We want to clarify the bifurcating situation by the following two figures in the case $0=p \neq q$. In order to take care of the rotational invariance of $\hat{u}(q) \in \mathbf{C}$, we superpose the real axis of $\hat{u}(q)$ on the $\hat{u}(0)$-axis. At the bifurcation points it will be clear from the context in which direction the branch bifurcates. (See Figures 2 and 3.)

REMARKS. (1) Note that in the theorem the case $\hat{w}(q)>\hat{w}(p)$ for $p \neq 0$, i.e., $(p p)(q q)-(p q)^{2}>0$, is covered by (i) and (ii) with $p$ and $q$ exchanged.

(2) By continuity we get from (5.10) and (5.13) the bifurcation conditions

$$
\begin{aligned}
& \partial \phi_{q}(\lambda,|\hat{u}(q)|)=\hat{w}(q) / \hat{w}(p) \quad \text { at } \lambda=\lambda_{q p}, \\
& \partial \phi_{0}(\lambda,|\hat{u}(0)|)=\hat{w}(0) / \hat{w}(q) \quad \text { at } \lambda=\lambda_{0 q} .
\end{aligned}
$$

We know from (2.47) that

$$
0 \leq \partial \phi_{0}(\lambda,|\hat{u}(0)|)<1 \text { for } \lambda \in\left(\lambda_{0},+\infty\right),
$$

where the upper and lower bounds are approached for $\lambda \searrow \lambda_{0}$ and $\lambda \rightarrow+\infty$, respectively. Similarly, by $(4.10)$,

$$
\frac{1}{2}<\partial \phi_{q}(\lambda,|\hat{u}(q)|)<1
$$

for $\lambda \in\left(\lambda_{q},+\infty\right)$, and again the bounds are approached for $\lambda \searrow \lambda_{q}$ and $\lambda \rightarrow+\infty$. But unfortunately, the functions $\partial \phi_{q}(\lambda,|\hat{u}(q)|)$ and $\partial \phi_{0}(\lambda,|\hat{u}(0)|)$ are, in general, not decreasing. Therefore, the sets

$$
\begin{aligned}
& \Delta_{q p}=\left\{\lambda>\lambda_{q}, \partial \phi_{q}(\lambda,|\hat{u}(q)|)<\hat{w}(q) / \hat{w}(p)\right\} \supseteq\left(\lambda_{q p},+\infty\right), \\
& \Delta_{0 q}=\left\{\lambda>\lambda_{0}, \partial \phi_{0}(\lambda,|\hat{u}(0)|)<\hat{w}(0) / \hat{w}(q)\right\} \supseteq\left(\lambda_{0 q},+\infty\right)
\end{aligned}
$$

may be composed by several nonconnected intervals. It is now easy to generalize the results of Theorem 5 , such that for each $\lambda \in \Delta_{q p}, \lambda \in \Delta_{0 q}$, respectively, there are $(p, q)$-secondary solutions, $(0, q)$-secondary solutions, respectively. Thus, if we have strict inclusions in (5.19) and (5.20), we get secondary bifurcating branches, which again vanish. Schematically, we get the bifurcation picture in Figure 4.

As a common phenomenon (see e.g. [7, Chapter II.11]), the appearance of the secondary bifurcations is followed by an exchange of stability. Here, we note this stability behavior only in terms of definitions (2.25)-(2.26). The results are consequences of more detailed stability investigations in $\S 8$.

THEOREM 6. Let the general assumptions of Theorem 5 hold.

(i) If $\frac{1}{2}>\hat{w}(q) / \hat{w}(p)>0$, then the p-primary branch is $p$-stable and $q$-stable, while the q-primary branch is $q$-stable but not $p$-stable.

(ii) If $1>\hat{w}(q) / \hat{w}(p)>\frac{1}{2}$, then the p-primary branch is again $p$-stable and $q$-stable, the q-primary branch is q-stable on $\left(\lambda_{q},+\infty\right)$, but at the bifurcations it 


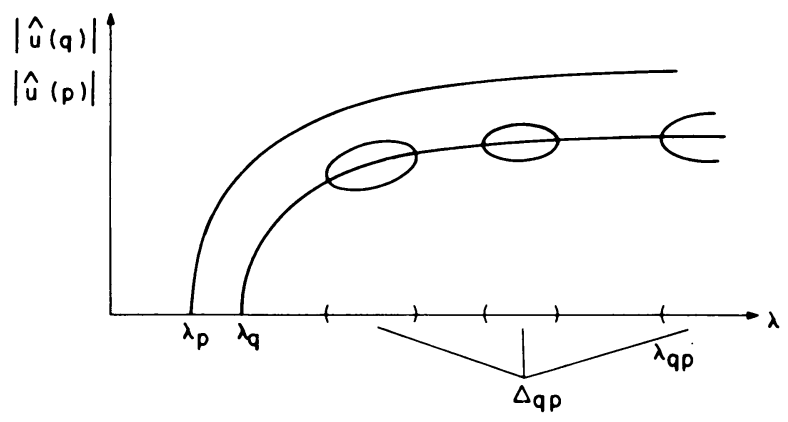

FIGURE 4

changes from a p-unstable or critical solution on $\left(\lambda_{q},+\infty\right) \backslash \Delta_{q p}$ to a $p$-stable solution on $\Delta_{q p}$.

(iii) If $p=0$ and $1>\hat{w}(0) / \hat{w}(q)>0$, then the q-primary branch is $q$-stable and 0-stable, the 0-primary branch is 0-stable, but it is q-unstable or critical on $\left(\lambda_{0},+\infty\right) \backslash \Delta_{0 q}$ and $q$-stable on $\Delta_{0 q}$.

6. An example: A secondary phase transition of first order. In this section we give an extension of some results from [2]. There, for different interaction potentials $J$, the equilibrium state in the thermodynamic limit for some mean-field models from statistical mechanics on the circle $\mathbf{T}$ are studied.

It is shown that in the ferromagnetic, but also in the antiferromagnetic case, there exist phase transitions of the equilibrium states. In particular, the phase transition for the antiferromagnetic circle is linked with a breaking of the continuous symmetry group $\mathbf{T}$. In the context of the present paper, the phase transitions of the ferromagnetic and antiferromagnetic circles correspond to the bifurcations of 0 -primary, respectively, $p$-primary, solutions of $(2.1)$ from the trivial solution $u \equiv 0$, which represents the paramagnetic state. The secondary solutions, which we have found in the last section, cannot, however, represent equilibrium states of the corresponding models of statistical mechanics, since the secondary solutions found are not stable. Nevertheless, in an indirect way, the secondary solutions are of physical relevance. Though they do not appear directly, they give rise to a secondary phase transition of first order.

The secondary bifurcation of the secondary solutions is linked with a change of the stability behavior of the primary solutions; in the words of Theorem 6(iii): if $\hat{w}(q)>\hat{w}(0)>0$, the 0 -primary solutions $u_{0}^{+/-}$are unstable on $\left(\lambda_{0},+\infty\right) \backslash \Delta_{0 q}$, but are stable for $\lambda \in \Delta_{0 q} \supseteq\left(\lambda_{0 q},+\infty\right)$, while the $p$-primary solutions are stable for all $\lambda>\lambda_{p}$. Therefore, for $\lambda \in \Delta_{0 q}$, both primary branches are stable. Now the equilibrium state has to make its choice between these two possible candidates by a variational principle. For $\lambda$ between $\lambda_{q}$ and $\lambda_{0}$ the equilibrium state will certainly be one of the $q$-primary solutions, since these are the only stable solutions there. By continuity, the equilibrium state will remain a $q$-primary solution even for values $\lambda$, which are little greater than $\lambda_{0}$. But for very large $\lambda$ it is possible that the newly stable 0 -primary solutions win the variational principle. If this is the case, there must be an intermediate value $\lambda^{*}$ where the equilibrium state jumps from a 
$q$-primary solution to a 0 -primary solution. We have a secondary phase transition of first order.

The following example shows that this phenomenon may really happen. In order to make things as easy as possible and to have a close connection to the representation in [2], we restrict ourselves to the case $d=1$, though the results hold for general dimension $d$.

At the sites $\alpha / n \in \mathbf{T}, \alpha=1, \ldots, n$, there are fixed magnetic spins $X_{\alpha}^{n}$. Without interaction, the $X_{\alpha}^{n}$ take independently the values +1 and -1 with probability $\frac{1}{2}$; i.e.,

$$
\rho_{0}=\left(\delta_{+1}+\delta_{-1}\right) / 2 .
$$

We let the interaction potential have the form

$$
J(s, t)=w(s-t)=1+2 b \cos (2 \pi q(s-t))
$$

with $q \in \mathbf{N}$, and

$$
1<b<\pi^{2} / 4 \text {. }
$$

The Hamiltonian of the interacting system is then given by

$$
H_{n}\left(X^{n}\right)=-\frac{1}{2 n} \sum_{\alpha_{1}, \alpha_{2}=1}^{n} J\left(\frac{\alpha_{1}}{n}, \frac{\alpha_{2}}{n}\right) X_{\alpha_{1}}^{n} X_{\alpha_{2}}^{n},
$$

and the common distribution of $\left(X_{\alpha}^{n}\right)_{\alpha=1, \ldots, n}$ is the Gibbs state to the Hamiltonian $H_{n}$ :

$$
\operatorname{Prob}_{n \beta}\left(X_{\alpha}^{n} \in d x_{\alpha}, \alpha=1, \ldots, n\right)=\frac{\exp \left(-\beta H_{n}(x)\right) \prod_{\alpha=1}^{n} \rho\left(d x_{\alpha}\right)}{Z_{n \beta}},
$$

where $x=\left(x_{1}, \ldots, x_{n}\right)$ and $Z_{n \beta}$ is the normalizing constant

$$
Z_{n \beta}=\int_{\mathbf{R}^{n}} \exp \left(-\beta H_{n}(x)\right) \prod_{\alpha=1}^{n} \rho\left(d x_{\alpha}\right) .
$$

In [2, Theorems 1.3 and 2.1] it is shown that in the thermodynamic limit the free energy $\psi(\beta)$ is given by the variational principle

$$
-\beta \psi(\beta):=\lim _{n \rightarrow \infty} n^{-1} \ln Z_{n \beta}=\sup _{f \in \mathcal{H}}[\beta F(f)-I(f)] .
$$

Here the functionals $F$ and $I$ are defined on $\mathscr{H}=L^{2}(\mathbf{T})$ by

$$
F(f)=\frac{1}{2} \iint_{\mathbf{T}^{2}} J(s, t) f(s) f(t) d s d t=\frac{1}{2}\langle f, w * f\rangle
$$

and

$$
I(f)=\int_{\mathbf{T}} i(f(t)) d t
$$

with

$$
i(u)= \begin{cases}{[(1+u) \ln (1+u)+(1-u) \ln (1-u)] / 2} & \text { for }|u| \leq 1 \\ +\infty & \text { for }|u|>1\end{cases}
$$

(See formulas (1.16)-(1.22) in [2].) 
By [2, Theorem 5.1] the supremum in (6.7) is always achieved, and any maximizing function $f$ satisfies the mean field equation

$$
i^{\prime}(f(t))=\beta\left(F^{\prime} f\right)(t) \quad \text { for almost all } t \in \mathbf{T} .
$$

In our example we have from (6.8) and (6.10) that

$$
\operatorname{th}^{-1}(f(t))=\beta \cdot w * f(t),
$$

or, equivalently, (2.1) with $\lambda=\beta$ and $g=\left(i^{\prime}\right)^{-1}=$ th (see also (2.10)).

Next, we make use of Fenchel's duality (see [2, Appendix C]).

$$
\sup _{f \in \mathcal{H}}[\beta F(f)-I(f)]=\sup _{f \in \mathcal{H}}\left[I^{*}(f)-(\beta F)^{*}(f)\right]
$$

where $I^{*}$ and $(\beta F)^{*}$ are the Legendre transforms of $I$ and $\beta F$, respectively. In our case we get, by $[2$, Lemma 3.6 and the remark thereafter],

$$
I^{*}(f)=\left(\Gamma^{*}\right)^{*}(f)=\Gamma(f)=\int \phi_{\rho_{0}}(f(t)) d t
$$

where $\phi_{\rho_{0}}$ is given in (2.9), and $\phi_{\rho_{0}}^{\prime}=g(2.10)$.

On the other hand, we find by easy calculations that

$$
\begin{aligned}
(\beta F)^{*}(f) & :=\sup _{h \in \mathcal{H}}\{\langle f, h\rangle-\beta F(h)\} \\
& =\left\{\begin{array}{cc}
\beta F\left(f_{0}\right) & \text { if } f=\beta w * f_{0} \text { for some } f_{0} \in \mathcal{H}, \\
+\infty & \text { otherwise. }
\end{array}\right.
\end{aligned}
$$

Note that (6.15) is well defined, since $f=\beta w * f_{1}=\beta w * f_{2}$ implies

$$
\beta F\left(f_{1}\right)=\frac{1}{2}\left\langle f_{1}, \beta w * f_{2}\right\rangle=\frac{1}{2}\left\langle f_{2}, \beta w * f_{2}\right\rangle=\beta F\left(f_{2}\right) .
$$

Thus, we can rewrite (6.7) as

$$
-\beta \psi(\beta)=\lim _{n \rightarrow \infty} n^{-1} \ln Z_{n}=\sup _{f \in \mathcal{H}}[\Gamma(\beta w * f)-\beta F(f)] .
$$

Now, if the maximum of $(6.16)$ is achieved at $f$, then $f$ has to satisfy the mean field equation, which is now written in the form

$$
\langle g\{\beta w * f\}, \beta w * h\rangle-\langle f, \beta w * h\rangle=0
$$

for all $h \in \mathcal{H}$. Note that by (6.15) we have reduced the variational principle to the space $w * \mathcal{H}$. But, moreover, $f$ must satisfy the second-order condition

$$
\left\langle g^{\prime}\{\beta w * f\} \cdot \beta w * h, \beta w * h\right\rangle-\langle h, \beta w * h\rangle \leq 0
$$

for all $h \in \mathcal{H}$. By the form (6.2) of $w,(6.18)$ implies, in particular (by calculations analogous to (4.18)),

$$
\beta \hat{w}(r) \int g^{\prime}\{\beta w * f(t)\} d t \leq 1
$$

for $r=0, q$. This is the stability condition (2.25) with $\leq$ instead of $<$. For $0<\beta \leq$ $1 / b=\beta_{q}$, the trivial solution $u \equiv 0$ is the only solution of $(6.17)$ and

$$
-\beta \psi(\beta)=0, \quad \beta \in(0,1 / b] \text {. }
$$

By (5.16) the bifurcation point $\beta_{0 q}$ for the $(0, q)$-secondary solutions satisfies

$$
b \beta_{0 q}\left(1-\operatorname{th}^{2}\left(\beta_{0 q} \hat{u}_{\beta_{0 q}}(0)\right)=1\right.
$$


and $\beta_{0 q}>\beta_{0}=1>\beta_{q}=1 / b$. For $\beta \in\left(\beta_{q}, 1\right)$ the $q$-primary solutions $u_{q}^{s}(4.7)$ are the only stable solutions, and

$$
-\beta \psi(\beta)=\beta b\left|\hat{u}_{\beta}(q)\right|^{2} / 2-\int i\left(\operatorname{th}\left(\beta b\left|\hat{u}_{\beta}(q)\right| 2 \cos (2 \pi q t)\right)\right) d t>0 .
$$

So we have a first phase transition at $\beta_{q}=1 / b$. The phase transition is of second order, since $\hat{u}_{\beta}(q) \rightarrow 0$ as $\beta \searrow \beta_{q}$. But for $\beta>\beta_{0 q}$ there are at least two different types of stable solutions: the $q$-primary and the 0 -primary solutions. For $\beta \rightarrow+\infty$ we find by $(2.46)$ with $\gamma=1$,

$$
\beta F\left(\equiv \hat{u}_{\beta}(0)\right)-I\left(\equiv \hat{u}_{\beta}(0)\right)=\beta \hat{u}_{\beta}(0)^{2} / 2-i\left(\hat{u}_{\beta}(0)\right) \approx \beta / 2,
$$

since $i$ is bounded by $\ln 2$ for $\left|\hat{u}_{\beta}(0)\right| \leq 1$, while

$$
\beta F\left(u_{q}^{s}(\beta, \cdot)\right)-I\left(u_{q}^{s}(\beta, \cdot)\right)=\beta b\left(\hat{u}_{\beta}(q)\right)^{2} / 2-I\left(u_{q}^{s}(\beta, \cdot)\right) \approx \beta b 2 / \pi^{2}
$$

by (4.9). Now (6.3) implies that (6.23) is greater than (6.24) for $\beta$ large enough. The maximum of $(6.16)$ is not attained any longer on the $q$-primary solutions. But by Theorem 5 the $q$-primary solutions do not have bifurcations. Therefore, there exists a $\beta^{*} \in(1,+\infty)$, where the maximum point jumps from a $q$-primary solution to another solution of (2.1). We have found a secondary phase transition of first order, as claimed at the beginning of the section. For $\beta$ large enough the new maximum is attained by a constant nontrivial solution, which corresponds to a ferromagnetic equilibrium state.

7. Secondary bifurcations for collinear $p, q$. As one may expect, the behavior of secondary bifurcations is different if $p$ and $q$ are collinear; i.e.,

$$
n_{1} p=n_{2} q \neq 0 \text { for some } n_{1}, n_{2} \in \mathbf{Z}
$$

with $\operatorname{gcd}\left(n_{1}, n_{2}\right)=1$. As in (3.9) we set

$$
r_{0}=p / n_{2}=q / n_{1} \in \mathbf{Z}^{d} .
$$

Of course, we assume that $w$ again satisfies condition (3.25), which by (3.10) can be rewritten as

$$
\hat{w}(r)=0 \quad \text { for all } r \in \begin{cases}\mathbf{Z} r_{0} \backslash\{ \pm p, \pm q\} & \text { if } n_{1} \cdot n_{2} \text { even } \\ (2 \mathbf{Z}+1) r_{0} \backslash\{ \pm p, \pm q\} & \text { if } n_{1} \cdot n_{2} \text { odd }\end{cases}
$$

and that

$$
\hat{w}(p)>\hat{w}(q)>0 .
$$

In the noncollinear case (3.25) implied condition (3.21) for $p$ and for $q$ (instead of $p$ ). Therefore, we could consider the $p$-primary and the $q$-primary branches in the last section. To guarantee this also in the collinear case, we must assume

$$
p \notin(2 \mathbf{Z}+1) q \text { and } q \notin(2 \mathbf{Z}+1) p .
$$

Now, we get the following result about secondary bifurcations which is proved in $\S 9$. The assumptions about $g$ from the beginning of $\S 5$ are still valid. 
THEOREM 7. Let $p, q \in \mathbf{Z}^{d}$ satisfy (7.1) and (7.5), and let (7.3) and (7.4) hold.

(i) There are never in $\mathcal{F}_{p q}$ bifurcations from the p-primary solutions.

(ii) If $\frac{1}{2} \geq \hat{w}(q) / \hat{w}(p)>0$ and $p \notin \mathbf{Z} q$, i.e., $n_{1} \neq 1$, then the branch of $q$-primary solutions does not have a secondary bifurcation in $\mathcal{F}_{p q}$.

(iii) If $1>\hat{w}(q) / \hat{w}(p)>\frac{1}{2}$ and $p \notin \mathbf{Z} q$, then

$$
\left(\lambda_{q},+\infty\right) \supseteq \Delta_{q p} \supseteq\left(\lambda_{q p},+\infty\right) \neq \varnothing
$$

with $\Delta_{q p}$ and $\lambda_{q p}$ from (5.19) and (5.10), respectively.

For $\lambda \in \Delta_{q p}$ there are the following branches of $(p, q)$-secondary solutions, which bifurcate from the q-primary solutions:

$$
\begin{aligned}
v_{1}(t)=g\left\{\lambda \hat{w}(p)\left|\hat{v}_{1}(p)\right| 2 \sin \left(2 \pi\left(p t+\left(\tau_{1}+j_{1}\right) / n_{1}+l_{1} / 2\right)\right)\right. \\
\left.+\lambda \hat{w}(q)\left|\hat{v}_{1}(q)\right| 2 \cos \left(2 \pi\left(q t+\left(\tau_{1}+k_{1}\right) / n_{2}+m_{1} / 2\right)\right)\right\}
\end{aligned}
$$

and

$$
\begin{aligned}
v_{2}(t)=g\left\{\lambda \hat{w}(p)\left|\hat{v}_{2}(p)\right| 2 \cos \left(2 \pi\left(p t+\left(\tau_{2}+j_{2}\right) / n_{1}+l_{2} / 2\right)\right)\right. \\
\left.+\lambda \hat{w}(q)\left|\hat{v}_{2}(q)\right| 2 \sin \left(2 \pi\left(q t+\left(\tau_{2}+k_{2}\right) / n_{2}+m_{2} / 2\right)\right)\right\},
\end{aligned}
$$

$t \in \mathbf{T}^{d}$, with the parameters $\tau_{1}, \tau_{2} \in \mathbf{T} ; j_{1}, j_{2} \in\left\{0, \ldots, n_{1}-1\right\} ; k_{1}, k_{2} \in\{0, \ldots$, $\left.n_{2}-1\right\} ; l_{1}, l_{2}, m_{1}, m_{2} \in\{0,1\}$.

(iv) If $p \in 2 q \mathbf{Z}$, i.e., $n_{1}=1$ and $n_{2}$ even, then there exists always a secondary bifurcation in $\mathcal{F}_{p q}$. It takes place at

$$
\begin{aligned}
\lambda_{q p}^{1}=\inf \left\{\lambda>\lambda_{q}, \lambda^{\prime} \hat{w}(q)\right. & \int g^{\prime}\left\{\lambda^{\prime} \hat{w}(q)|\hat{u}(q)| 2 \cos (2 \pi s)\right\} \\
& \left.\times\left(1-\cos \left(4 \pi n_{2} s\right)\right) d s<\hat{w}(q) / \hat{w}(p) \text { for all } \lambda^{\prime}>\lambda\right\}
\end{aligned}
$$

with

$$
\lambda_{q} \leq \lambda_{q p}^{1}<+\infty .
$$

For $\lambda \in\left(\lambda_{q p}^{1},+\infty\right)$, we have branches of $(p, q)$-secondary solutions of the form $v_{1}$ from (7.7) (with $n_{1}=1$ ).

REMARKS. (1) Mutans mutandum, remark (2) after Theorem 5 also holds here: On some intervals there may be bifurcating branches of the forms $v^{1}$ or $v^{2}$ described above, which appear, disappear, and reappear according to the conditions appearing in (5.19) and (7.9), respectively. We define

$$
\begin{aligned}
\Delta_{q p}^{1}=\left\{\lambda>\lambda_{q}, \lambda \hat{w}(q) \int g^{\prime}\left\{\lambda \hat{w}(q)|\hat{u}(q)| 2 \cos \left(2 \pi n_{1} s\right)\right\}\right. & \\
& \left.\times\left(1-\cos \left(4 \pi n_{2} s\right)\right) d s<\hat{w}(q) / \hat{w}(p)\right\},
\end{aligned}
$$

$$
\begin{aligned}
\Delta_{q p}^{2}=\left\{\lambda>\lambda_{q}, \lambda \hat{w}(q) \int g^{\prime}\left\{\lambda \hat{w}(q)|\hat{u}(q)| 2 \sin \left(2 \pi n_{1} s\right)\right\}\right. & \\
& \left.\times\left(1+\cos \left(4 \pi n_{2} s\right)\right) d s<\hat{w}(q) / \hat{w}(p)\right\} .
\end{aligned}
$$


If $n_{2} \notin n_{1} \mathbf{Z}$, then by Proposition $1(\mathrm{i})$, we can cancel the last cosine term in (7.11) and (7.12) and get

$$
\Delta_{q p}=\Delta_{q p}^{1}=\Delta_{q p}^{2}
$$

So we may have $\Delta_{q p}^{1} \supseteq\left(\lambda_{q p}^{1},+\infty\right) \neq \varnothing$ with strict inclusion. In the case $p \in 2 q \mathbf{Z}$, i.e., $n_{1}=1$ and $n_{2}$ even, $\Delta_{q p}^{2}$ is always a bounded, possibly empty region in $\mathbf{R}^{+}$. If $\lambda \in \Delta_{q p}^{2} \neq \varnothing$, we have secondary bifurcating solutions of the form $v_{2}$ from (7.8) with $n_{1}=1$. However, these solutions disappear again as $\lambda \rightarrow+\infty$.

(2) In (7.7) and (7.8) let us disregard the rotation group $\tau \in \mathbf{T}$ for a moment; i.e., put $\tau=0$. Then since $g$ is invertible, $v^{1}$ and $v^{2}$ represent $8 \cdot n_{1} \cdot n_{2}$ different secondary solutions if $n_{1} \cdot n_{2}$ is odd. If $n_{1} \cdot n_{2}$ is even, let $n_{1}$ be even, for example; then the parameters $i_{1}=n_{1} / 2, k_{1}=1$, and $i_{1}=k_{1}=0$ give the same solution. Similarly for $i_{2}$ and $k_{2}$. Therefore, we have only $4 \cdot n_{1} \cdot n_{2}$ different secondary solutions if $n_{1} \cdot n_{2}$ is even. This fact corresponds to result (3.10).

(3) We refer to the end of $\S 9$ for some considerations concerning the stability of the solutions in the collinear case.

8. The associated dynamical system for noncollinear $p, q$. We continue to let $g$ satisfy the additional conditions from the beginning of $\S 5$, let $p$ and $q$ be noncollinear in the sense of (5.3), and let (3.25) hold with $\hat{w}(p)>0, \hat{w}(q)>0$. To prove the results from Theorems 5 and 6 , we need a good knowledge of the fixed point problem for $z=\left(z_{1}, z_{2}\right) \in \mathbf{R}^{2}$ :

$$
z=\phi(z)=\left(\phi_{1}\left(z_{1}, z_{2}\right), \phi_{2}\left(z_{1}, z_{2}\right)\right)
$$

where

$$
\begin{array}{r}
\phi_{1}(x, y)=\int g\left\{\lambda \hat{w}(p) x\left[\begin{array}{c}
1 \\
2 \cos (2 \pi p t)
\end{array}\right]\right. \\
+\lambda \hat{w}(q) y 2 \cos (2 \pi q t)\} \cos (2 \pi p t) d t
\end{array}
$$

$$
\phi_{2}(x, y)=\int g\{\cdots\} \cos (2 \pi q t) d t
$$

where we have in $\{\cdots\}$ the same argument as in (8.2). Of course, all fixed points of $\phi$ are contained in

$$
\Omega=\bigcap_{n \geq 0} \overline{\phi^{n}\left(\mathbf{R}^{2}\right)}
$$

where $\phi^{n}=\phi \circ \cdots \circ \phi$ ( $n$ times $)$. We shall see that here $\Omega$ is exactly the set of all fixed points: There are no periodic orbits or more complicated variant limit sets. It turns out that $\overline{\phi\left(\mathbf{R}^{2}\right)}$ is a very nice compact convex set, independent of $\lambda, \hat{w}(p)$ and $\hat{w}(q)$. 
THEOREM 8.

$$
\overline{\phi\left(\mathbf{R}^{2}\right)}=\left\{\begin{array}{c}
\left\{(\gamma x, \gamma y) ;|y| \leq \frac{2}{\pi} \cos \left(\frac{\pi x}{2}\right),|x| \leq 1\right\} \quad \text { for } p=0 \neq q, \\
\left\{\left(\gamma x_{1}, \gamma x_{2}\right) ;\left|x_{i}\right|=\frac{8}{\pi} \int_{0}^{1 / 4} \frac{\mu \sin ^{2}(2 \pi r)}{\sqrt{1-\mu^{2} \cos ^{2}(2 \pi r)}} d r\right. \\
\left|x_{3-i}\right| \leq \frac{8}{\pi} \int_{0}^{1 / 4} \sqrt{1-\mu^{2} \cos ^{2}(2 \pi r)} d r \\
0 \leq \mu \leq 1, i=1,2\} \\
\text { for }(p p)(q q)-(p q)^{2}>0 .
\end{array}\right.
$$

PROOF. First assume $p=0 \neq q$. For $0 \neq|x| \leq 1$ we set $m(x)=1 / \sin (\pi x / 2)$ and check that

$$
\operatorname{sign}\{1+m(x) \cos (2 \pi r)\}=\operatorname{sign}(x) \cdot\left(2 \cdot 1_{[0,(1+x) / 4]}(|r|)-1\right)
$$

for $|r| \leq \frac{1}{2}$ and $4|r| \neq 1+x, x \neq 0$. Then

$$
\begin{aligned}
\lim _{\alpha \rightarrow \infty} \phi & \left.\phi \frac{\alpha x}{\hat{w}(0) \lambda}, \pm \frac{\alpha x m(x)}{2 \lambda \hat{w}(q)}\right) \\
= & \gamma\left(\int_{\mathbf{T}^{d}} \operatorname{sign}\{x(1 \pm m(x) \cos (2 \pi q t))\} d t,\right. \\
= & \int_{\mathbf{T}^{d}} \operatorname{sign}\left\{x \left(1 \pm m(x) \operatorname{sign}(x)\left(\int_{-1 / 2}^{+1 / 2} \operatorname{sign}\{1+m(x) \cos (2 \pi r)\} d r,\right.\right.\right. \\
= & \pm \cdot\left(x, \pm \frac{2}{\pi} \sin \left(\frac{2 \pi(1+x)}{4}\right)\right) \\
= & \left(\gamma x, \pm \frac{2 \gamma}{\pi} \cos \left(\frac{\pi x}{2}\right)\right) .
\end{aligned}
$$

Since $\overline{\phi\left(\mathbf{R}^{2}\right)}$ is simply connected, we have

$$
\overline{\phi\left(\mathbf{R}^{2}\right)} \supseteq\{(\gamma x, \gamma y),|y| \leq(2 / \pi) \cos (\pi x / 2)\} \text {. }
$$

If we had strict inclusion in the last line, there would exist $\left(x_{0}, y_{0}\right) \in \mathbf{R}^{2}$ with

$$
\phi\left(x_{0}, y_{0}\right)=\left(\gamma \phi_{1}, \gamma \phi_{2}\right)
$$

and

$$
\phi_{2}=(2 / \pi) \cos \left(\pi \phi_{1} / 2\right) .
$$

The curve $y=(2 / \pi) \cos (\pi x / 2)$ has in $\left(\phi_{1}, \phi_{2}\right)$ the outer normal direction $\bar{n}=$ $\left(\sin \left(\pi \phi_{1} / 2\right), 1\right)$. For $\alpha \in \mathbf{R}^{+}$set

$$
h(\alpha)=\bar{n} \cdot \phi\left(x_{0}+(\alpha / \lambda \hat{w}(0)) \sin \left(\pi \phi_{1} / 2\right), y_{0}+\alpha / 2 \lambda \hat{w}(q)\right) .
$$


Then, for all $\alpha \in \mathbf{R}^{+}$,

$$
\begin{aligned}
\frac{d}{d \alpha} h(\alpha)=\int g^{\prime}\left\{\lambda \hat{w}(0) x_{0}+\alpha \sin \left(\pi \phi_{1} / 2\right)+\left(\lambda 2 \hat{w}(q) y_{0}+\alpha\right) \cos (2 \pi q t)\right\} & \\
& \times\left(\sin \left(\pi \phi_{1} / 2\right)+\cos (2 \pi q t)\right)^{2} d t>0 .
\end{aligned}
$$

But, as in (8.7),

$$
\begin{aligned}
\lim _{\alpha \rightarrow \infty} h(\alpha)=\gamma \cdot \bar{n} \cdot\left(\int \operatorname{sign}\left\{\sin \left(\pi \phi_{1} / 2\right)+\cos (2 \pi q t)\right\} d t,\right. \\
\left.\int \operatorname{sign}\left\{\sin \left(\pi \phi_{1} / 2\right)+\cos (2 \pi q t)\right\} \cos (2 \pi q t) d t\right) \\
=\gamma \cdot \bar{n} \cdot\left(\phi_{1},(2 / \pi) \cos \left(\pi \phi_{1} / 2\right)\right)=h(0)
\end{aligned}
$$

gives a contradiction.

For $(p p)(q q)=(p q)^{2}>0$ and $0 \leq \mu \leq 1$, we calculate

$$
\begin{aligned}
\lim _{\alpha \rightarrow \infty} \phi(\alpha \mu / 2 \lambda \hat{w}(p), \pm \alpha / 2 \lambda \hat{w}(q)) \\
=\gamma\left(\begin{array}{l}
\int \operatorname{sign}\{\mu \cos (2 \pi p t) \pm \cos (2 \pi q t)\} \cos (2 \pi p t) d t \\
\int \operatorname{sign}\{\mu \cos (2 \pi p t) \pm \cos (2 \pi q t)\} \cos (2 \pi q t) d t
\end{array}\right) .
\end{aligned}
$$

Set $B_{q p}=\left\{(r, s), r=t p, s=t q, t \in \mathbf{T}^{d}\right\}$, and the last expression equals

$$
\begin{aligned}
& 2 \gamma /\left|B_{p q}\right|\left(\begin{array}{l}
\int_{B_{p q}} 1_{\{\mu \cos (2 \pi r) \pm \cos (2 \pi s)>0\}} \cos (2 \pi r) d r d s \\
\int_{B_{p q}}^{1} 1_{\{\mu \cos (2 \pi r) \pm \cos (2 \pi s)>0\}} \cos (2 \pi s) d r d s
\end{array}\right) \\
&= 2 \gamma\left(\begin{array}{l}
\left.\int_{-1 / 2}^{+1 / 2} \int_{-1 / 2}^{+1 / 2} 1_{\{|s| \lessgtr \arccos (\mp \mu \cos (2 \pi r)) / 2 \pi\}} \cos (2 \pi r) d r d s\right) \\
\int_{-1 / 2}^{+1 / 2} \int_{-1 / 2}^{-1 / 2} 1_{\{|s| \lessgtr \arccos (\mp \mu \cos (2 \pi r)) / 2 \pi\}} \cos (2 \pi s) d r d s
\end{array}\right) \\
&=\gamma 2 / \pi\left(\begin{array}{l}
\int_{-1 / 2}^{+1 / 2} \pm \arccos (\mp \mu \cos (2 \pi r)) \cos (2 \pi r) d r \\
\int_{-1 / 2}^{+1 / 2} \pm \sin \arccos (\mp \mu \cos (2 \pi r)) d r
\end{array}\right) \\
&=\gamma 4 / \pi\left(\begin{array}{l}
\int_{0}^{1 / 4}(\pi-2 \arccos (\mu \cos (2 \pi r)) \cos (2 \pi r)) d r \\
\int_{0}^{1 / 4} \pm 2 \sin \arccos _{0}(\mu \cos (2 \pi r)) d r
\end{array}\right) \\
&=\gamma 8 / \pi\left(\begin{array}{l}
\int_{0}^{1 / 4} \frac{\mu \sin ^{2}(2 \pi r)}{\sqrt{1-\mu^{2} \cos ^{2}(2 \pi r)}} d r \\
\pm \int_{0}^{1 / 4} \sqrt{1-\mu^{2} \cos ^{2}(2 \pi r)} d r
\end{array}\right)
\end{aligned}
$$

by partial integration. For $\alpha \rightarrow-\infty$ or $\mu=1 / \mu^{\prime} \in[1,+\infty)$ we get the other 
boundary points of the right side of (8.5). Thus $\overline{\phi\left(\mathbf{R}^{2}\right)}$ contains the right side of (8.5). The converse inclusion is shown by a similar argument as in the first part of the proof.

REMARK. Thanks to Theorem 8 , it is sufficient to regard $\phi$ as acting on the universal set $\overline{\phi\left(\mathbf{R}^{2}\right)}$, which is independent of $\lambda, \hat{w}(p)$, and $\hat{w}(q)$. Moreover, the geometric form of $\overline{\phi\left(\mathbf{R}^{2}\right)}$ can be used to analyse the behavior of the equilibrium states and their phase transitions. In particular, the ground states can be nicely discussed with the help of the set $\overline{\phi\left(\mathbf{R}^{2}\right)}$. See also [10, $\S \S \mathrm{VII}$ and VIII], where the ground states of a spin-glass model are studied in detail.

The essential step for determining the fixed points of $\phi$ is to analyse the fixed points of the components $\phi_{1}$ and $\phi_{2}$ separately. We note the following easy facts:

(i) $(x, y)$ is a fixed point of $\phi$ if and only if $x$ is a fixed point of $\phi_{1}(\cdot, y)$ and $y$ is a fixed point of $\phi_{2}(x, \cdot)$.

(ii) 0 is a fixed point for $\phi_{1}(\cdot, y)$ and $\phi_{2}(x, \cdot)$ for all $y \in \mathbf{R}, x \in \mathbf{R}$, respectively.

(iii) If $(x, y)$ is a stable fixed point of $\phi$, then so is $x$ for $\phi_{1}(\cdot, y)$ and $y$ for $\phi_{2}(x, \cdot)$.

However, the converse of the last statement is not true, as we shall see later.

In contrast to $g$, the functions $\phi_{1}(\cdot, y)$ and $\phi_{2}(x, \cdot)$ need not be concave on $(0, \infty)$, in general. Instead of concavity, we use the following result.

LEMMA. Under the conditions from the beginning of this section, $\phi_{1}(x, y)$ is strictly increasing and odd in $x$ but even in $y$. For $x \in \mathbf{R} \backslash\{0\}$ fixed, $\left|\phi_{1}(x, \cdot)\right|$ is strictly decreasing in $|y|$ with

$$
\lim _{|y| \rightarrow \infty}\left|\phi_{1}(x, y)\right|=0
$$

The same assertions hold for $\phi_{2}$ with $x$ and $y$ exchanged.

PROOF. Similarly to the first equations in (8.8)-(8.9), we get

$$
\begin{gathered}
\phi_{1}(x, y)=\int_{-1 / 2}^{+1 / 2} \int_{-1 / 2}^{+1 / 2} g\left\{\lambda \hat{w}(p) x\left[\begin{array}{l}
1 \\
2 \cos (2 \pi r)
\end{array}\right]+\lambda \hat{w}(q) y 2 \cos (2 \pi s)\right\} \\
\times\left[\begin{array}{l}
1 \\
\cos (2 \pi r)
\end{array}\right] d r d s \\
\phi_{2}(x, y)=\int_{-1 / 2}^{+1 / 2} \int_{-1 / 2}^{+1 / 2} g\{\cdots\} \cos (2 \pi s) d r d s
\end{gathered}
$$

again repeating the argument in $\{\cdots\}$ from (8.11). The first assertions are then easily verified by the properties of $g$. For the second assertion we can assume 
$x>0, y>0$. Then

$$
\begin{aligned}
& \partial_{y} \phi_{1}(x, y)=2 \hat{w}(q)\left[\begin{array}{c}
1 / \hat{w}(0) \\
1 / 2 \hat{w}(p)
\end{array}\right] \partial_{x} \phi_{2}(x, y) \\
& =2 \lambda \hat{w}(q) \int_{-1 / 2}^{+1 / 2} \int_{-1 / 2}^{+1 / 2} g^{\prime}\left\{\lambda \hat{w}(p) x\left[\begin{array}{l}
1 \\
2 \cos (2 \pi r)
\end{array}\right]+\lambda \hat{w}(q) y 2 \cos (2 \pi s)\right\} \\
& \times\left[\begin{array}{l}
1 \\
\cos (2 \pi r)
\end{array}\right] \cos (2 \pi s) d r d s \\
& =\lambda \hat{w}(q) \int_{-1 / 2}^{+1 / 2} \int_{-1 / 2}^{+1 / 2}\left(g^{\prime}\left\{\lambda \hat{w}(p) x\left[\begin{array}{l}
1 \\
2 \cos (2 \pi r)
\end{array}\right]+\lambda \hat{w}(q) y 2 \cos (2 \pi s)\right\}\right. \\
& \left.\left.-g^{\prime}\left\{\lambda \hat{w}(p) x\left[\begin{array}{l}
1 \\
2 \cos (2 \pi r)
\end{array}\right] \begin{array}{c}
-\lambda \hat{w}(q) y 2 \cos (2 \pi s)\}) \\
1 \\
<0,
\end{array}\right] \begin{array}{c}
1 \\
\cos (2 \pi r)
\end{array}\right]\right) \\
& \cos (2 \pi s) d r d s
\end{aligned}
$$

since the integrand is a.e. negative, as seen by cases.

Finally, $\phi_{1}(x, y) \rightarrow_{|y| \rightarrow \infty} 0$ and $\phi_{2}(x, y) \rightarrow_{|x| \rightarrow \infty} 0$ follow from (8.11) and (8.12).

By the lemma, we can now describe the fixed points of $\phi_{1}$ and $\phi_{2}$ separately. Recall the definition of $\lambda_{p}, \lambda_{q}$ from (2.36) or (4.6) and of $|\hat{u}(p)|,|\hat{u}(q)|$ from (2.37) or (4.8).

THEOREM 9. For $\lambda \in\left(0, \lambda_{p}\right], x=0$ is the only fixed point of $\phi_{1}(\cdot, y)$ for all $y$. For $\lambda \in\left(\lambda_{p},+\infty\right)$ there exists a unique, positive, symmetric, continuously differentiable function

$$
\psi_{1}:(-|\hat{u}(p)|,+|\hat{u}(p)|) \rightarrow(0, \infty)
$$

with

$$
\phi_{1}\left(x, \psi_{1}(x)\right)=\phi_{1}\left(x,-\psi_{1}(x)\right)=x
$$

for all $x \in(-|\hat{u}(p)|,+|\hat{u}(p)|)$. We have

$$
\lim _{|x| \rightarrow|\hat{u}(p)|} \psi_{1}(x)=0
$$

$\psi_{1}(0)>0$ is uniquely determined by

$$
\lambda \hat{w}(p) \int g^{\prime}\left\{\lambda \hat{w}(q) \psi_{1}(0) 2 \cos (2 \pi q t)\right\} d t=1 .
$$

Similar assertions hold for $\phi_{2}(x, \cdot)$ by a function

$$
\psi_{2}:(-|\hat{u}(q)|,+|\hat{u}(q)|) \rightarrow(0, \infty),
$$


where $p$ and $q, x$ and $y$ are exchanged everywhere. Here, $\psi_{2}(0)$ is uniquely determined by

$$
\lambda \hat{w}(q) \int g^{\prime}\left\{\lambda \hat{w}(p) \psi_{2}(0)\left[\begin{array}{c}
1 \\
2 \cos (2 \pi p t)
\end{array}\right]\right\} d t=1
$$

REMARK. We want to point out that the strict concavity of $g$ is essential for Theorem 9. If $g$ were linear on some intervals, then as in the remarks following Theorems 1 and 3, we would not have uniqueness for the values of $\psi_{1}$ satisfying (8.14). The points of a whole interval would then satisfy these equations, and equally the set of fixed points of $\phi$ in $(0, \infty)^{2}$ could then have a two-dimensional subset.

Proof. For $\lambda \in\left(0, \lambda_{p}\right]$ and $x>0$, we get by the lemma and the definition of $\lambda_{p}$ that

$$
0<\phi_{1}(x, y) \leq \phi_{1}(x, 0)=\int g\left\{\lambda \hat{w}(p) x\left[\begin{array}{c}
1 \\
2 \cos (2 \pi p t)
\end{array}\right]\right\} \cos (2 \pi p t) d t<x
$$

for all $y \in \mathbf{R}$. $\phi_{1}$ being odd in $x, x=0$ is thus the only fixed point of $\phi_{1}(\cdot, y)$ for all $y$.

Now let $\lambda \in\left(\lambda_{p},+\infty\right) . \phi_{1}(x, 0)=\phi_{p}(\lambda, x)$ from (2.44) or (4.2) is strictly concave in $x>0$ with $\left|\hat{u}_{\lambda}(p)\right|$ as the unique positive fixed point. Thus

$$
\begin{array}{ll}
\left|\phi_{1}(x, 0)\right|>|x| & \text { for }|x| \in(0,|\hat{u}(p)|), \\
\left|\phi_{1}(x, 0)\right|<|x| & \text { for }|x| \in(|\hat{u}(p)|,+\infty) .
\end{array}
$$

Hence, by the second assertion of the lemma, exactly for $|x| \in(0,|\hat{u}(p)|)$, there exists a unique $y=\psi_{1}(x)>0$ with

$$
\phi_{1}(x, y)=\phi_{1}(x,-y)=x \text {. }
$$

Since $\phi_{1}$ is odd in $x, \psi_{1}$ is symmetric in $x$. By the implicit function theorem $\psi_{1}$ is continuously differentiable and

$$
\frac{d}{d x} \psi_{1}(x)=\frac{1-\partial_{x} \phi_{1}\left(x, \psi_{1}(x)\right)}{\partial_{y} \phi_{1}\left(x, \psi_{1}(x)\right)}
$$

where $\partial_{x} \phi_{1}$ (resp. $\partial_{y} \phi_{1}$ ) denote the partial derivatives of $\phi_{1}$ with respect to $x$ (resp. $y)$. Next, we show that $\psi_{1}$ is bounded on $(0,|\hat{u}(p)|)$. Set

$$
\tilde{g}^{\prime}(v)=\sup \left\{g^{\prime}\{v+2 \lambda \hat{w}(p) x\} ; 0 \leq|x| \leq|\hat{u}(p)|\right\} .
$$

Since $\int \tilde{g}^{\prime}\{\lambda \hat{w}(q) y 2 \cos (2 \pi q t)\} d t \rightarrow|y| \rightarrow \infty 0$, we find $y_{0}>0$ with

$$
\lambda \hat{w}(p) \int \tilde{g}^{\prime}\left\{\lambda \hat{w}(q) y_{0} 2 \cos (2 \pi q t)\right\}\left[\begin{array}{l}
1 \\
2 \cos ^{2}(2 \pi p t)
\end{array}\right] d t<1
$$




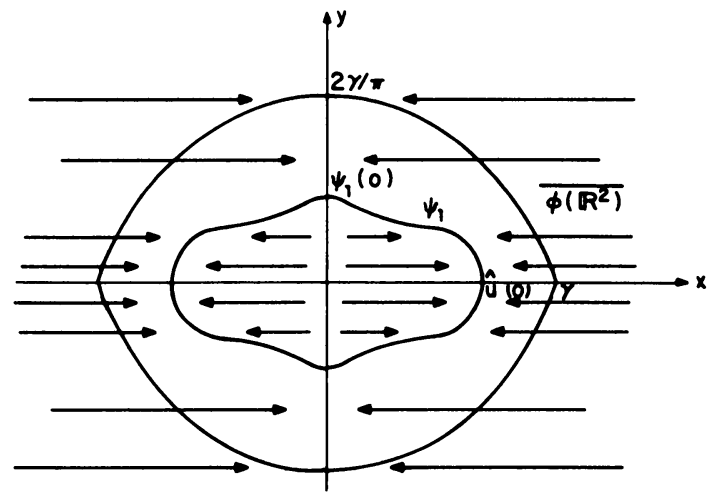

FIGURE 5. The function $\psi_{1}$ and the action of $\phi_{1}(\cdot, y), y$ fixed, $p=0 \neq q$.

Therefore, by the mean value theorem

$$
\begin{aligned}
\phi_{1}\left(x, y_{0}\right) & =\int g\left\{\lambda \hat{w}(p) x\left[\begin{array}{l}
1 \\
2 \cos (2 \pi p t)
\end{array}\right]\right. \\
& \left.+\lambda \hat{w}(q) y_{0} 2 \cos (2 \pi q t)\right\} \cos (2 \pi p t) d t \\
& \leq \lambda \hat{w}(p)|x| \int \tilde{g}^{\prime}\left\{\lambda \hat{w}(q) y_{0} 2 \cos (2 \pi q t)\right\}\left[\begin{array}{c}
1 \\
2 \cos ^{2}(2 \pi p t)
\end{array}\right] d t \\
& <|x|
\end{aligned}
$$

for all $|x| \leq|\hat{u}(p)|$, and

$$
\psi_{1}(x)<y_{0} \quad \text { for all }|x|<|\hat{u}(p)| \text {. }
$$

Since by definition of $|\hat{u}(p)|, \phi_{1}( \pm|\hat{u}(p)|, 0)= \pm|\hat{u}(p)|$, the boundedness of $\psi_{1}$ implies (8.15). Since $g^{\prime}$ is strictly decreasing on $\mathbf{R}^{+}$, so is $\partial_{x} \phi_{1}(0, \cdot)$. If $\partial_{x} \phi_{1}(x, y) \lessgtr 1$ for $(x, y)=\left(0, y_{0}\right)$, this inequality also holds in a neighborhood $U$ of $\left(0, y_{0}\right)$ in $(0, \infty)^{2}$. Since $\phi_{1}(0, y)=0$, we find $\phi_{1}(x, y) \lessgtr x$ for all $(x, y) \in U$, and $\left(0, y_{0}\right)$ cannot be an accumulation point of $\left(x, \psi_{1}(x)\right)$. Hence, $\psi_{1}(0)$ is uniquely determined by

$$
\partial_{x} \phi_{1}\left(0, \psi_{1}(0)\right)=1
$$

which is equivalent to (8.16), since $p$ and $q$ are noncollinear. The analogue of (8.21) for $\psi_{2}$ is

$$
\frac{d}{d y} \psi_{2}(y)=\frac{1-\partial_{y} \phi_{2}\left(\psi_{2}(y), y\right)}{\partial_{x} \phi_{2}\left(\psi_{2}(y), y\right)}
$$

For $p=0 \neq q$ we get the picture of $\psi_{1}$ shown in Figure 5. The arrows indicate the action of $\phi_{1}(\cdot, y)$ for $y$ fixed.

The proof of Theorem 9 and fact (ii) preceding the lemma give the following complete description of the fixed points of $\phi$. 
THEOREM 10.

$$
\begin{aligned}
F= & {\left[\{0\} \times \mathbf{R} \cup\left\{\left(x, \pm \psi_{1}(x)\right),|x| \leq|\hat{u}(p)|\right\}\right] } \\
& \cap\left[\mathbf{R} \times\{0\} \cup\left\{\left( \pm \psi_{2}(y), y\right),|y| \leq|\hat{u}(q)|\right\}\right]
\end{aligned}
$$

is the set of all fixed points of $\phi$.

To formulate the following relations between $\psi_{1}(0)$ and $|\hat{u}(q)|$ and between $\psi_{2}(0)$ and $|\hat{u}(p)|$, we recall the dependence on $\lambda$ of $\psi_{1}$ and $\psi_{2}$, though not made explicit, and the definition of $\Delta_{q p}$ and $\Delta_{0 q}$ in (5.19)-(5.20). For $\lambda \leq \lambda_{p}$ we set, for convenience, $|\hat{u}(p)|=0$ and $\psi_{1}=0$, and similarly $|\hat{u}(q)|=0$ and $\psi_{2}=0$ for $\lambda \leq \lambda_{q}$.

THEOREM 11. (i) If $\frac{1}{2}>\hat{w}(q) / \hat{w}(p)>0$, then

$$
\left.\begin{array}{l}
|\hat{u}(p)|>\psi_{2}(0) \\
\psi_{1}(0)>|\hat{u}(q)|
\end{array}\right\} \quad \text { for } \lambda \in\left(\lambda_{p},+\infty\right) .
$$

(ii) If $1>\hat{w}(q) / \hat{w}(p)>\frac{1}{2}$, then

$$
\begin{gathered}
|\hat{u}(p)|>\psi_{2}(0) \quad \text { for } \lambda \in\left(\lambda_{p},+\infty\right), \\
\psi_{1}(0)>|\hat{u}(q)| \quad \text { for } \lambda \in\left(\lambda_{p}, \lambda_{q}\right),
\end{gathered}
$$

but

$$
\psi_{1}(0)<|\hat{u}(q)| \text { exactly for } \lambda \in \Delta_{q p} \supseteq\left(\lambda_{q p},+\infty\right) .
$$

(iii) If $p=0$ and $1>\hat{w}(0) / \hat{w}(q)>0$, then

$$
\begin{gathered}
\psi_{1}(0)<|\hat{u}(q)| \quad \text { for } \lambda \in\left(\lambda_{q},+\infty\right), \\
|\hat{u}(0)|<\psi_{2}(0) \quad \text { for } \lambda \in\left(\lambda_{q}, \lambda_{0}\right),
\end{gathered}
$$

but

$$
|\hat{u}(0)|>\psi_{2}(0) \text { exactly for } \lambda \in \Delta_{0 q} \supseteq\left(\lambda_{0 q},+\infty\right) .
$$

PROOF. First, remark that (8.32) holds trivially since $\psi_{1}(0)>0=|\hat{u}(q)|$ for $\lambda \in\left(\lambda_{p}, \lambda_{q}\right)$. Similarly for (8.35). By $(2.47)$ or (4.10) we find

$$
\lambda \hat{w}(q) \int g^{\prime}\left\{\lambda \hat{w}(p)|\hat{u}(p)|\left[\begin{array}{l}
1 \\
2 \cos (2 \pi p t)
\end{array}\right]\right\} d t \in \hat{w}(q) / \hat{w}(p) \cdot(0,1)
$$

for $\lambda>\lambda_{p}$, and

$$
\lambda \hat{w}(p) \int g^{\prime}\{\lambda \hat{w}(q)|\hat{u}(q)| 2 \cos (2 \pi q t)\} d t \in \hat{w}(p) / \hat{w}(q) \cdot\left(\frac{1}{2}, 1\right)
$$

for $\lambda>\lambda_{p}$. Thus, for $\lambda>\lambda_{p},(8.17)$ implies $\left|\hat{u}_{\lambda}(p)\right|>\psi_{2}(0)$ if $\hat{w}(q) / \hat{w}(p)<1$, i.e., (8.29) and (8.31); and for $\lambda>\lambda_{q},(8.16)$ implies $\psi_{1}(0)>|\hat{u}(q)|$ if $\hat{w}(p) / 2 \hat{w}(q)>1$, i.e., $(8.30)$, or, for $p=0, \psi_{1}(0)<|\hat{u}(q)|$ for $\hat{w}(0) / \hat{w}(q)<1$, i.e., (8.34). But if $1 \in \hat{w}(p) / \hat{w}(q) \cdot\left(\frac{1}{2}, 1\right)$, then by definition $(5.19)$,

$$
\lambda \hat{w}(p) \int g^{\prime}\{\lambda \hat{w}(q)|\hat{u}(q)| 2 \cos (2 \pi q t)\}<1
$$




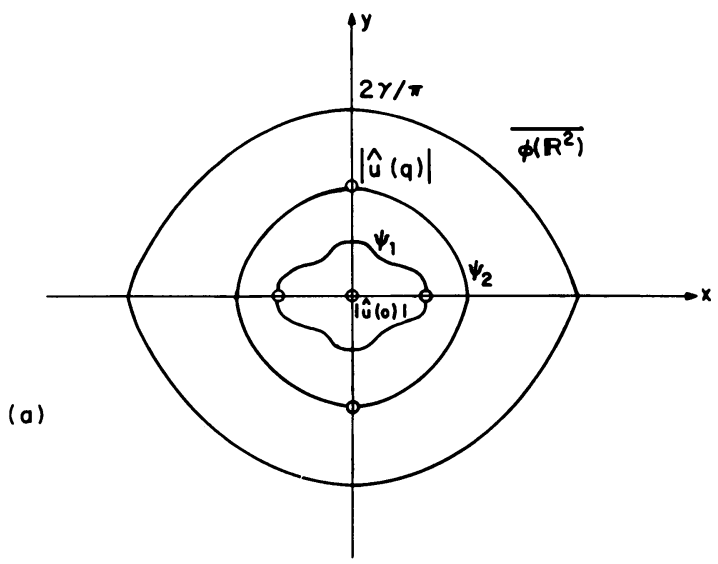

(b)

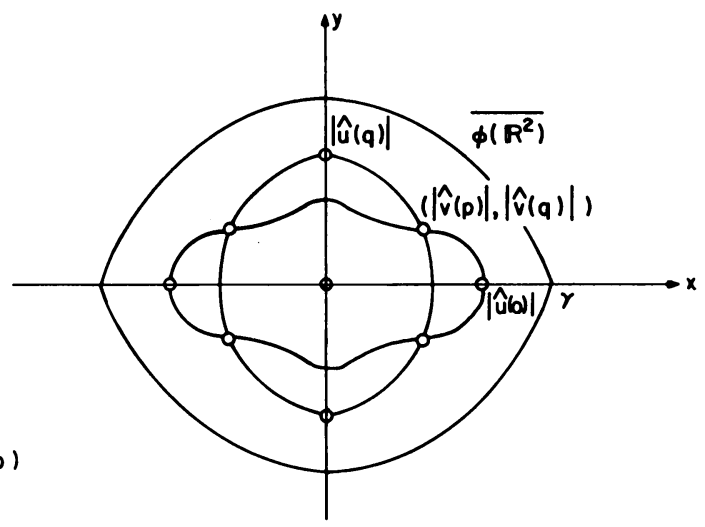

FIGURE 6. The functions $\psi_{1}(x)$ and $\psi_{2}(y)$ for $p=0 \neq q, \hat{w}(q)>\hat{w}(q)$ $>\hat{w}(0)>0$, and $\lambda \in\left(\lambda_{0}, \lambda_{0 q}, \lambda_{0 q}\right)$ in (a) (resp. $\lambda>\lambda_{0 q}$ in (b)). The fixed points of $\phi$ are noted by a small circle.

if and only if $\lambda \in \Delta_{q p} \neq \varnothing$, such that (8.16) implies (8.33). Similarly, for $p=0$ and $1 \in \hat{w}(q) / \hat{w}(0) \cdot(0,1)$, we have, by $(5.20)$,

$$
\lambda \hat{w}(q) g^{\prime}\{\lambda|\hat{u}(0)| \hat{w}(0)\}<1
$$

if and only if $\lambda \in \Delta_{0 q}$, i.e., (8.36).

REMARKS. (i) Remark (i) after Theorem 5 also applies here.

(ii) The proof of (8.29) verifies just the branching condition: there are no bifurcations on the $p$-primary branch of solutions into the direction of $(p, q)$-secondary solutions. Conversely, (8.32) and (8.33) prove that indeed a secondary bifurcation occurs on the branch of $q$-primary solutions, and similarly in case (iii) with $p=0$ and $q$ exchanged. In case (i) there does not exist a secondary bifurcation on either branch of primary solutions. Nevertheless, to know, in this case, if there are no $(p, q)$-secondary solutions at all, one has to compute the functions $\psi_{1}$ and $\psi_{2}$ and to see if their graphs intersect as in Figure 6.

We like to note that Figure 6 is a little optimistic, since for general $g$ one cannot prove without additional assumptions that the graphs of $\psi_{1}$ and $\psi_{2}$ have no intersection in case $\lambda<\lambda_{0 q}(\mathrm{a})$, and exactly one intersection point in $(0, \infty)^{2}$ in 
case $\lambda>\lambda_{0 q}$ (b), though this is what we expect in most examples. The results of Theorems 10 and 11 have the following immediate consequence according to fact (i) preceding the lemma.

COROLlaRY. If (ii) $1>\hat{w}(q) / \hat{w}(p)>\frac{1}{2}$ and $\lambda \in \Delta_{q p}$, or if (iii) $p=0 \neq q, 1>$ $\hat{w}(0) / \hat{w}(q)>0$ and $\lambda \in \Delta_{0 q}$, then there exists at least one fixed point of $\phi$ in $(0, \infty)^{2} \cap \phi\left(\mathbf{R}^{2}\right)$. By $(|\hat{v}(p)|,|\hat{v}(q)|)$ we denote that fixed point of $\phi$ in $(0, \infty)^{2}$, for which $|\hat{v}(q)|$ in case (ii) (resp. $|\hat{v}(0)|$ in case (iii)) is maximal.

The results of this section enable us to prove Theorem 5 of $\S 5$.

PROOF OF THEOREM 5. (i) If $\frac{1}{2}>\hat{w}(q) / \hat{w}(p)>0$, then the expression in (8.37) is less than $\frac{1}{2}$ for all $\lambda>\lambda_{p}$, and the expression in (8.38) is greater than 1 for all $\lambda>\lambda_{q}$. So in $\mathcal{F}_{p q}$ there are no secondary bifurcations either on the $p$-primary, or on the $q$-primary branch.

(ii) If $1>\hat{w}(q) / \hat{w}(p)>\frac{1}{2}$, then the expression in (8.37) is still less than 1 for all $\lambda>\lambda_{p}$, and in $\mathcal{F}_{p q}$ there is no secondary bifurcation on the $p$-primary branch. But (4.11) for $q$ shows that $\lambda_{q p}$, defined in (5.10), is finite and $\Delta_{q p} \supseteq\left(\lambda_{q p},+\infty\right) \neq$ $\varnothing$. $\lambda_{q}<\lambda_{q p}$ follows from $|\hat{u}(q)| \rightarrow \lambda_{\lambda \lambda_{q}} 0$. By continuity (8.31)-(8.33) and the corollary show that there exists a bifurcation of $(p, q)$-secondary solutions of the form (5.12), which branches off the $q$-primary solution and exists for all $\lambda \in \Delta_{q p}$.

(iii) Let $p=0 \neq q$ and $1>\hat{w}(0) / \hat{w}(q)>0$. The expression in (8.38) is less than 1 for all $\lambda>\lambda_{q}$, and in $\mathcal{F}_{0 q}$ there is no secondary bifurcation on the branch of $q$-primary solutions. Here, (2.48) yields $\lambda_{0 q}<+\infty$, and (8.35)-(8.36) show the existence of a secondary bifurcation in $\mathcal{F}_{0 q}$ on the branch of nontrivial constant solutions. The $(0, q)$-secondary solutions are of the form (5.12) with $p=0$ and exist for all $\lambda \in \Delta_{0 q}$. By (2.43) we have $\lambda_{0}<\lambda_{0 q}$.

We finish this section by describing the stability properties of the fixed points of $\phi$. We use the following terminology:

DEFinition. A fixed point $z$ of $\phi$ is called stable if all eigenvalues $\mu_{i}$ of the linearization $\partial \phi$ of $\phi$ at $z$ have modulus less than 1: $\left|\mu_{i}\right|<1$ for all eigenvalues $\mu_{i} . z$ is called a hyperbolic fixed point if for some eigenvalues $\mu_{i_{0}}, \mu_{i_{1}}$ of $\partial \phi$ at $z$ we have $\left|\mu_{i_{0}}\right|<1,\left|\mu_{i_{1}}\right|>1$, and $\left|\mu_{i}\right| \neq 1$ for all other eigenvalues. $z$ is called (totally) unstable if $\left|\mu_{i}\right|>1$ for all eigenvalues $\mu_{i}$ of $\partial \phi$ at $z$. $z$ is called critical if $\left|\mu_{i}\right|=1$ for at least one eigenvalue $\mu_{i}$ of $\partial \phi$ at $z$.

THEOREM 12. The fixed points of $\phi$ have the following properties:

(i) If $\frac{1}{2}>\hat{w}(q) / \hat{w}(p)>0$, then

$$
\begin{aligned}
& (0,0) \text { is }\left\{\begin{array}{l}
\text { stable for } \lambda \in\left(0, \lambda_{p}\right), \\
\text { hyperbolic for } \lambda \in\left(\lambda_{p}, \lambda_{q}\right), \\
\text { unstable for } \lambda \in\left(\lambda_{q},+\infty\right) ;
\end{array}\right. \\
& ( \pm|\hat{u}(p)|, 0) \text { is stable for } \lambda \in\left(\lambda_{p},+\infty\right) ;
\end{aligned}
$$

(ii) If $1>\hat{w}(q) / \hat{w}(p)>\frac{1}{2}$, then (8.41) and (8.42) hold again, but

$$
(0, \pm|\hat{u}(q)|) \text { is }\left\{\begin{array}{l}
\text { hyperbolic or critical for } \lambda \in\left(\lambda_{q},+\infty\right) \backslash \Delta_{q p} \\
\text { stable for } \lambda \in \Delta_{q p}
\end{array}\right.
$$




$$
( \pm|\hat{v}(p)|, \pm|\hat{v}(q)|) \text { is hyperbolic or critical for all } \lambda \in \Delta_{q p} .
$$

(iii) If $p=0 \neq q$ and $1>\hat{w}(0) / \hat{w}(q)>0$, then

$$
\begin{gathered}
(0,0) \text { is }\left\{\begin{array}{l}
\text { stable for } \lambda \in\left(0, \lambda_{q}\right), \\
\text { hyperbolic for } \lambda \in\left(\lambda_{q}, \lambda_{0}\right), \\
\text { unstable for } \lambda \in\left(\lambda_{0},+\infty\right) ;
\end{array}\right. \\
(0, \pm|\hat{u}(q)|) \text { is stable for } \lambda \in\left(\lambda_{q},+\infty\right) ; \\
( \pm|\hat{u}(0)|, 0) \text { is }\left\{\begin{array}{l}
\text { hyperbolic or critical for } \lambda \in\left(\lambda_{0},+\infty\right) \backslash \Delta_{0 q} \\
\text { stable for } \lambda \in \Delta_{0 q} ;
\end{array}\right. \\
( \pm|\hat{v}(0)|, \pm|\hat{v}(q)|) \text { is hyperbolic or critical for } \lambda \in \Delta_{0 q} .
\end{gathered}
$$

REMARK. $\pm|\hat{v}(p)|$ is a stable fixed point of $\phi_{1}(\cdot, \pm|\hat{v}(q)|)$, and $\pm|\hat{v}(q)|$ is a stable fixed point of $\phi_{2}( \pm|\hat{v}(p)|, \cdot)$, since they lie on the graphs of $\psi_{1}$ and $\psi_{2}$, respectively, which represent stable fixed points for $\phi_{1}(\cdot, y)$ and $\phi_{2}(x, \cdot)$, respectively. This implies that the corresponding solution (5.12) is $p$-stable and $q$-stable. However, with regard to $\phi,( \pm|\hat{v}(p)|, \pm|\hat{v}(q)|)$ is not stable. See also the remark at fact (iii) preceding the lemma.

PROOF. The linearization of $\phi$ is given by

$$
\partial \phi=\left(\begin{array}{ll}
\partial_{x} \phi_{1} & \partial_{y} \phi_{1} \\
\partial_{x} \phi_{2} & \partial_{y} \phi_{2}
\end{array}\right)
$$

where

$$
\begin{gathered}
\partial_{x} \phi_{1}(x, y)=\lambda \hat{w}(p) \int g^{\prime}\left\{\lambda \hat{w}(p) x\left[\begin{array}{l}
1 \\
2 \cos (2 \pi p t)
\end{array}\right]+\lambda \hat{w}(q) y 2 \cos (2 \pi p t)\right\} \\
\times\left[\begin{array}{l}
1 \\
2 \cos ^{2}(2 \pi p t)
\end{array}\right] d t \\
\partial_{y} \phi_{2}(x, y)=\lambda \hat{w}(q) \int g^{\prime}\{\cdots\} 2 \cos ^{2}(2 \pi q t) d t
\end{gathered}
$$

and

$$
\partial_{y} \phi_{1}(x, y)=2 \hat{w}(q)\left[\begin{array}{c}
1 / \hat{w}(0) \\
1 / 2 \hat{w}(p)
\end{array}\right] \partial_{x} \phi_{2}(x, y)
$$

is given in (8.13). By a calculation similar to the first equations in (8.8)-(8.9), we get

$$
\partial_{y} \phi_{1}(x, y)=\partial_{x} \phi_{2}(x, y)=0 \quad \text { if } x=0 \text { or } y=0 .
$$

(8.41) and (8.46) are obvious from the definition of $\lambda_{p}, \lambda_{q}$ in (2.36) or (4.6). (2.38) or the concavity of $\phi_{q}(\lambda, z)$ show

$$
\partial_{x} \phi_{1}( \pm|\hat{u}(p)|, 0)=\frac{\partial}{\partial z} \phi_{p}(\lambda,|\hat{u}(p)|) \in(0,1) \text { for } \lambda \in\left(\lambda_{p},+\infty\right)
$$

and

$$
\partial_{y} \phi_{2}(0, \pm|\hat{u}(q)|)=\frac{\partial}{\partial z} \phi_{q}(\lambda,|\hat{u}(q)|) \in(0,1) \text { for } \lambda \in\left(\lambda_{q},+\infty\right)
$$


By (8.37), (8.38), and the noncollinearity of $p, q$, we get

$$
\begin{aligned}
\partial_{y} \phi_{2}( \pm|\hat{u}(p)|, 0) & =\lambda \hat{w}(q) \int g^{\prime}\left\{ \pm \lambda \hat{w}(p)|\hat{u}(p)|\left[\begin{array}{l}
1 \\
2 \cos (2 \pi p t)
\end{array}\right]\right\} 2 \cos ^{2}(2 \pi q t) d t \\
& \in \hat{w}(q) / \hat{w}(p) \cdot(0,1)
\end{aligned}
$$

and

$$
\partial_{x} \phi_{1}(0, \pm|\hat{u}(q)|) \in \hat{w}(p) / \hat{w}(q) \cdot\left(\frac{1}{2}, 1\right)
$$

Thus, if $\hat{w}(q) / \hat{w}(p)<1$ in case (i) or (ii), then $( \pm|\hat{u}(p)|, 0)$ is stable for $\lambda \in\left(\lambda_{p},+\infty\right)$. If $\hat{w}(p) / \hat{w}(q)>2$ in case (i), then $(0, \pm|\hat{u}(q)|)$ is hyperbolic, and if $\hat{w}(0) / \hat{w}(q)<1$ in case (iii), then it is stable for $\lambda \in\left(\lambda_{q},+\infty\right)$. If $\hat{w}(p) / \hat{w}(q) \in(1,2)$ in case (ii), then by (5.19) and (4.3) we obtain

$$
\partial_{x} \phi_{1}(0, \pm|\hat{u}(q)|)=\frac{\hat{w}(p)}{\hat{w}(q)} \partial \phi_{q}(\lambda,|\hat{u}(q)|)<1 \quad \text { iff } \quad \lambda \in \Delta_{q p}
$$

while in case (iii) with $\hat{w}(0) / \hat{w}(q)<1$ by $(5.20)$,

$$
\partial_{y} \phi_{2}( \pm|\hat{u}(0)|, 0)=\frac{\hat{w}(q)}{\hat{w}(0)} \partial \phi_{0}(\lambda,|\hat{u}(0)|)<1 \quad \text { iff } \quad \lambda \in \Delta_{0 q}
$$

This shows (8.44) and (8.48). For assertions (8.45) and (8.49), we have to calculate the eigenvalues

$$
\mu_{1 / 2}=\left(\partial_{x} \phi_{1}+\partial_{y} \phi_{2}\right) / 2 \pm\left[\left(\partial_{x} \phi_{1}+\partial_{y} \phi_{2}\right)^{2} / 4+\partial_{y} \phi_{1} \partial_{x} \phi_{2}-\partial_{x} \phi_{1} \partial_{y} \phi_{2}\right]^{1 / 2}
$$

at $( \pm|\hat{v}(p)|, \pm|\hat{v}(q)|)$. The maximality condition in the corollary says that in case (ii) the graph $\left\{\left(\psi_{2}(y), y\right) ; y \in(|\hat{v}(q)|,|\hat{u}(q)|)\right\}$ lies above the graph $\left\{\left(x, \psi_{1}(x)\right) ; x \in\right.$ $(0,|\hat{v}(p)|)\}$, while in case (iii) the graph $\left\{\left(x, \psi_{1}(x)\right) ; x \in(|\hat{v}(p)|,|\hat{u}(p)|)\right\}$ lies above $\left\{\left(\psi_{2}(y), y\right) ; y \in(0,|\hat{v}(q)|)\right\}$ (see Figure 6). Both cases imply that

$$
\left|\psi_{1}^{\prime}(|\hat{v}(p)|)\right| \leq 1 /\left|\psi_{2}^{\prime}(|\hat{v}(q)|)\right| .
$$

By (8.21) and (8.27) this shows that at $(|\hat{v}(p)|,|\hat{v}(q)|)$,

$$
0 \leq\left|\left(1-\partial_{x} \phi_{1}\right)\left(1-\partial_{y} \phi_{2}\right)\right| \leq \partial_{y} \phi_{1} \partial_{x} \phi_{2} .
$$

Assume first that $\left(1-\partial_{x} \phi_{1}\right)\left(1-\partial_{y} \phi_{2}\right) \geq 0$. Then in (8.54) we have

$$
\partial_{y} \phi_{1} \partial_{x} \phi_{2}-\partial_{x} \phi_{1} \partial_{y} \phi_{2} \geq 1-\left(\partial_{x} \phi_{1}+\partial_{y} \phi_{2}\right)
$$

such that with $a=\left(\partial_{x} \phi_{1}+\partial_{y} \phi_{2}\right) / 2>0$, we get $\mu_{1} \geq a+\left(a^{2}+1-2 a\right)^{1 / 2}=$ $a+|1-a| \geq 1$, and $\mu_{2} \leq a-|1-a| \leq 1$.

If, on the other hand, $\left(1-\partial_{x} \phi_{1}\right)\left(1-\partial_{y} \phi_{2}\right)<0$-i.e., $0<\partial_{x} \phi_{1}<1<\partial_{y} \phi_{2}$ or $0<\partial_{y} \phi_{2}<1<\partial_{x} \phi_{1}$-then, since

$$
\partial_{y} \phi_{1} \partial_{x} \phi_{2}=\left[\begin{array}{l}
\hat{w}(0) \\
2 \hat{w}(p)
\end{array}\right] \frac{\left(\partial_{y} \phi_{1}\right)^{2}}{2 \hat{w}(q)} \geq 0
$$

we get

$$
\begin{aligned}
& \mu_{1} \geq\left(\partial_{x} \phi_{1}+\partial_{y} \phi_{2}\right) / 2+\left|\partial_{x} \phi_{1}-\partial_{y} \phi_{2}\right| / 2=\max \left(\partial_{x} \phi_{1}, \partial_{y} \phi_{2}\right)>1 \\
& \mu_{2} \leq\left(\partial_{x} \phi_{1}+\partial_{y} \phi_{2}\right) / 2-\left|\partial_{x} \phi_{1}-\partial_{y} \phi_{2}\right| / 2=\min \left(\partial_{x} \phi_{1}, \partial_{y} \phi_{2}\right)<1
\end{aligned}
$$


To finish the proof of (8.45) and (8.49), we need only show that $\mu_{2} \geq 0$. But at $(|\hat{v}(p)|,|\hat{v}(q)|)$

$$
\begin{array}{r}
0 \leq \partial_{y} \phi_{1} \partial_{x} \phi_{2} \leq\left[\begin{array}{l}
2 \\
4
\end{array}\right] \lambda^{2} \hat{w}(p) \hat{w}(q) \times\left(\int g ^ { \prime } \left\{\lambda \hat{w}(p)|\hat{v}(p)|\left[\begin{array}{l}
1 \\
2 \cos (2 \pi p t)
\end{array}\right]\right.\right. \\
+\lambda \hat{w}(q)|\hat{v}(q)| 2 \cos (2 \pi q t)\} \\
\times|\cos (2 \pi p t)| \cdot|\cos (2 \pi q t)| d t)^{2}
\end{array}
$$

$\leq \partial_{x} \phi_{1} \partial_{y} \phi_{2}$

such that

$$
\begin{aligned}
\mu_{2} & =\left(\partial_{x} \phi_{1}+\partial_{y} \phi_{2}\right) / 2-\left(\left(\partial_{x} \phi_{1}-\partial_{y} \phi_{2}\right)^{2} / 4+\partial_{y} \phi_{1} \partial_{x} \phi_{2}\right)^{1 / 2} \\
& \geq a-|a|=0 .
\end{aligned}
$$

This completes the proof of Theorem 12.

The proof of Theorem 6 is now an immediate consequence of Theorem 12. We only note that for primary solutions, the linearization $\partial \phi$ has diagonal form by (8.53). The definition of $p$ - or $q$-stability is then by (4.14)-(4.15), and the noncollinearity of $p, q$ equivalent to the fact that $\partial_{x} \phi_{1}$ (resp. $\partial_{y} \phi_{2}$ ) is less than 1 .

9. The dynamical system for collinear $p, q$. We assume the collinearity conditions (7.1) and (7.5) for $p, q$, and (7.3) and (7.4) for the function $w$. In order to get in $\mathcal{F}_{p q}$ a secondary bifurcation from the $p$-primary solutions, one of the following bifurcation conditions must be satisfied:

$$
\begin{gathered}
1=\lambda \hat{w}(q) \int g^{\prime}\{\lambda \hat{w}(p) 2 \operatorname{Re}(\hat{u}(p) \exp (2 \pi i p t))\} 2 \cos ^{2}(2 \pi q t) d t \\
=\lambda \hat{w}(q) \int_{-1 / 2}^{1 / 2} g^{\prime}\left\{\lambda \hat{w}(p)|\hat{u}(p)| 2 \cos \left(2 \pi n_{2} s+\arg \hat{u}_{\lambda}(p)\right)\right\} \\
\times\left(1+\cos \left(4 \pi n_{1} s\right)\right) d s
\end{gathered}
$$

or

$$
\begin{aligned}
1=\lambda \hat{w}(q) \int_{-1 / 2}^{1 / 2} g^{\prime}\left\{\lambda \hat { w } ( p ) | \hat { u } ( p ) | 2 \operatorname { c o s } \left(2 \pi n_{2} s+\right.\right. & \left.\left.\arg \hat{u}_{\lambda}(p)\right)\right\} \\
& \times\left(1-\cos \left(4 \pi n_{1} s\right)\right) d s
\end{aligned}
$$

But we claim that the right expressions of (9.1) or (9.2) are always less than $\hat{w}(q) / \hat{w}(p)<1$. To verify this, set $\mu=2 \lambda \hat{w}(p)|\hat{u}(p)|>0$ and $\alpha=\arg \hat{u}(p)$ for $\lambda>\lambda_{p}$. First consider the case $n_{1} \notin n_{2}$ Z. By Proposition 1(i) we have

$$
\int g^{\prime}\left\{\mu \cos \left(2 \pi n_{2} s+\alpha\right)\right\} \cos \left(4 \pi n_{1} s\right) d s=0,
$$

and (4.3) and (4.10) yield

$$
\lambda \hat{w}(q) \int g^{\prime}\left\{\mu \cos \left(2 \pi n_{2} s+\alpha\right)\right\} d s \in \hat{w}(q) / \hat{w}(p)\left(\frac{1}{2}, 1\right)
$$


which proves our claim in this case. On the other hand, assume $n_{1}=l \cdot n_{2} \in$ $n_{2} \mathbf{Z}, l>1$. We note first that for all $\beta \in \mathbf{T}$ and $z \in\left(0, \frac{1}{2}\right)$

$$
\begin{aligned}
\int_{-z}^{z} & {[\cos (2 \pi s) \pm \cos (2 \pi(l s-\beta))] d s } \\
& =(1 / \pi l)[l \sin (2 \pi z) \pm \sin (2 \pi l z) \cos (2 \pi \beta)] \\
& \geq(1 / \pi l)[l \sin (2 \pi z)-|\sin (2 \pi l z)|]>0
\end{aligned}
$$

Since $g^{\prime}$ is decreasing on $\mathbf{R}^{+}$, we can define $\int d g^{\prime}(y)$ as a Lebesgue-Stieltjes integral on $\mathbf{R}^{+}$with

$$
\int_{a}^{b} d g^{\prime}(y)<0 \text { for all } 0 \leq a<b
$$

and

$$
g^{\prime}\{\mu|\cos (\pi s)|\}=g^{\prime}(0)+\int_{0}^{\mu} 1_{[y / \mu, 1]}(|\cos (\pi s)|) d g^{\prime}(y) .
$$

Then by (9.5)-(9.7),

$$
\begin{aligned}
\int_{-1 / 2}^{+1 / 2} & g^{\prime}\left\{\mu \cos \left(2 \pi n_{2} s+\alpha\right)\right\}\left[\cos \left(4 \pi n_{2} s+2 \alpha\right) \pm \cos \left(4 \pi n_{1} s\right)\right] d s \\
\quad= & \int_{-1 / 2}^{+1 / 2} g^{\prime}\{\mu|\cos (\pi s)|\}[\cos (2 \pi s) \pm \cos (2 \pi l s-2 l \alpha)] d s \\
& =\int_{0}^{\mu} d g^{\prime}(y) \int_{-\arccos (y / \mu) / \pi}^{\arccos (y / \mu) / \pi}[\cos (2 \pi s) \pm \cos (2 \pi l s-2 l \alpha)] d s<0
\end{aligned}
$$

or, by (4.13),

$$
\begin{aligned}
0<\lambda \hat{w}(q) \int_{-1 / 2}^{+1 / 2} g^{\prime}\left\{\mu \cos \left(2 \pi n_{2} s+\alpha\right)\right\} & \quad \times\left[2 \sin ^{2}\left(2 \pi n_{2} s+\alpha\right)-\left(1 \pm \cos \left(4 \pi n_{1} s\right)\right)\right] d s \\
= & \frac{\hat{w}(q)}{\hat{w}(p)}-\lambda \hat{w}(q) \int_{-1 / 2}^{+1 / 2} g^{\prime}\left\{\mu \cos \left(2 \pi n_{2} s+\alpha\right)\right\}\left(1 \pm \cos \left(4 \pi n_{1} s\right)\right) d s
\end{aligned}
$$

which proves our claim, following (9.2). Therefore, under the assumptions of Theorem 7 , there exists in $\mathcal{F}_{p q}$ no bifurcation from the $p$-primary branch of solutions.

To prove the existence of secondary bifurcations from the $q$-primary solutions, we use the same technique as in $\S 8$. Here we look for nondegenerate fixed points $(x, y), x \neq 0 \neq y$, of the following pair of operators:

$$
\phi^{1}(x, y)=\left(\phi_{1}^{1}(x, y), \phi_{2}^{1}(x, y)\right) \quad \text { and } \quad \phi^{2}(x, y)=\left(\phi_{1}^{2}(x, y), \phi_{2}^{2}(x, y)\right)
$$

where

$$
\begin{aligned}
\phi_{1}^{1}(x, y) & =\int g\{\lambda \hat{w}(p) x 2 \sin (2 \pi p t)+\lambda \hat{w}(q) y 2 \cos (2 \pi q t)\} \sin (2 \pi p t) d t \\
& =\int_{-1 / 2}^{+1 / 2} g\left\{\lambda \hat{w}(p) x 2 \sin \left(2 \pi n_{2} s\right)+\lambda \hat{w}(q) y 2 \cos \left(2 \pi n_{1} s\right)\right\} \sin \left(2 \pi n_{2} s\right) d s
\end{aligned}
$$


and similarly

$$
\begin{array}{r}
\phi_{2}^{1}(x, y)=\int_{-1 / 2}^{+1 / 2} g\left\{\lambda \hat{w}(p) x 2 \sin \left(2 \pi n_{2} s\right)+\lambda \hat{w}(q) y 2 \cos \left(2 \pi n_{1} s\right)\right\} \\
\times \cos \left(2 \pi n_{1} s\right) d s \\
\phi_{1}^{2}(x, y)=\int_{-1 / 2}^{+1 / 2} g\left\{\lambda \hat{w}(p) x 2 \cos \left(2 \pi n_{2} s\right)+\lambda \hat{w}(q) y 2 \sin \left(2 \pi n_{1} s\right)\right\} \\
\times \cos \left(2 \pi n_{2} s\right) d s \\
\phi_{2}^{2}(x, y)=\int_{-1 / 2}^{+1 / 2} g\left\{\lambda \hat{w}(p) x 2 \cos \left(2 \pi n_{2} s\right)+\lambda \hat{w}(q) y 2 \sin \left(2 \pi n_{1} s\right)\right\} \\
\times \sin \left(2 \pi n_{1} s\right) d s .
\end{array}
$$

For the pairs $\left(\phi_{1}^{1}, \phi_{2}^{1}\right)$ and $\left(\phi_{1}^{2}, \phi_{2}^{2}\right)$ we get the same results as in the lemma and Theorem 9 of $\S 8$.

THEOREM 13. The functions $\phi_{1}^{1}$ and $\phi_{1}^{2}$ are strictly increasing and odd in $x$ but even in $y$. For $x \neq 0$ they are strictly decreasing in $|y|$ with

$$
\lim _{|y| \rightarrow \infty}\left|\phi_{1}^{1}(x, y)\right|=\lim _{|y| \rightarrow \infty}\left|\phi_{1}^{2}(x, y)\right|=0 .
$$

For $\lambda \in\left(0, \lambda_{p}\right], x=0$ is the only fixed point of $\phi_{1}^{1}(\cdot, y)$ and $\phi_{1}^{2}(\cdot, y)$ for all $y$, while for $\lambda \in\left(\lambda_{p},+\infty\right)$ there exist unique, positive, symmetric, continuously differentiable functions $\psi_{1}^{1}$ and $\psi_{1}^{2}$ on $(-|\hat{u}(p)|,+|\hat{u}(p)|)$ with

$$
\phi_{1}^{1}\left(x, \pm \psi_{1}^{1}(x)\right)=\phi_{1}^{2}\left(x, \pm \psi_{1}^{2}(x)\right)=x
$$

for all $x \in(-|\hat{u}(p)|,+|\hat{u}(p)|)$, and

$$
\lim _{|x| \rightarrow\left|\hat{u}_{\lambda}(p)\right|} \psi_{1}^{i}(x)=0, \quad i=1,2 .
$$

The same facts hold for $\phi_{2}^{1}$ and $\phi_{2}^{2}$ with functions $\psi_{2}^{1}$ and $\psi_{2}^{2}$ on $(-|\hat{u}(q)|,+|\hat{u}(q)|)$ if we exchange $x$ and $y$ and $p$ and $q$ everywhere. At zero $\psi_{1}^{1}$ and $\psi_{1}^{2}$ are uniquely determined by the equations

$$
\begin{aligned}
& 1=\lambda \hat{w}(p) \int_{-1 / 2}^{+1 / 2} g^{\prime}\left\{\lambda \hat{w}(q) \psi_{1}^{1}(0) 2 \cos \left(2 \pi n_{1} s\right)\right\}\left(1-\cos \left(4 \pi n_{2} s\right)\right) d s \\
& 1=\lambda \hat{w}(p) \int_{-1 / 2}^{+1 / 2} g^{\prime}\left\{\lambda \hat{w}(q) \psi_{1}^{2}(0) 2 \sin \left(2 \pi n_{1} s\right)\right\}\left(1+\cos \left(4 \pi n_{2} s\right)\right) d s
\end{aligned}
$$

Analogous equations determine $\psi_{1}^{2}(0)$ and $\psi_{2}^{2}(0)$ uniquely.

The proof of this theorem follows the same lines as those of the lemma and Theorem 9 in $\S 8$. (8.13) is now replaced by

$$
\begin{aligned}
& \partial_{y} \phi_{1}^{1}(x, y)=\hat{w}(q) / \hat{w}(p) \partial_{x} \phi_{2}^{1}(x, y) \\
&=\lambda \hat{w}(q) \int_{-1 / 2}^{+1 / 2}\left[g ^ { \prime } \left\{\lambda \hat{w}(p) x 2 \sin \left(2 \pi n_{2} s\right)+\right.\right.\left.\lambda \hat{w}(q) y 2 \cos \left(2 \pi n_{1} s\right)\right\} \\
&\left.-g^{\prime}\left\{-\lambda \hat{w}(p) x 2 \sin \left(2 \pi n_{2} s\right)+\lambda \hat{w}(q) y 2 \cos \left(2 \pi n_{1} s\right)\right\}\right] \\
& \times \sin \left(2 \pi n_{2} s\right) \cos \left(2 \pi n_{1} s\right) d s
\end{aligned}
$$


which is less than 0 if $x>0$ and $y>0$. Similarly to (8.24), the boundedness of $\psi_{1}^{1}$ follows from

$$
\phi_{1}^{1}(x, y) \leq \lambda \hat{w}(p)|x| \int \tilde{g}^{\prime}\left\{\lambda \hat{w}(q) y 2 \cos \left(2 \pi n_{1} s\right)\right\}\left(1-\cos \left(4 \pi n_{2} s\right)\right) d s,
$$

which is less than $|x|$ if $y$ is only large enough. Here, $\tilde{g}^{\prime}$ is taken from (8.22).

For the following result, recall the definitions of $\Delta_{q p}, \Delta_{q p}^{1}$, and $\Delta_{q p}^{2}$ from (5.19), and (7.11), (7.12), respectively.

ThEOREM 14. (i) Assume $p \notin q \mathbf{Z}$. Then

$$
\Delta_{q p}^{1}=\Delta_{q p}^{2}=\Delta_{q p} \supseteq\left(\lambda_{q p},+\infty\right) \neq \varnothing \quad \text { iff } \quad 1>\hat{w}(q) / \hat{w}(p)>1 / 2 .
$$

(ii) If $p \in 2 q \mathbf{Z}$, then for all $\hat{w}(p)>\hat{w}(q)>0$,

$$
\Delta_{q p}^{1} \supseteq\left(\lambda_{q p}^{1},+\infty\right) \neq \varnothing,
$$

but $\Delta_{q p}^{2}$ is a bounded (possibly empty) region in $\mathbf{R}^{+}$.

(iii) For $i=1,2$ we have

$$
\begin{aligned}
& |\hat{u}(p)|>\psi_{2}^{i}(0) \quad \text { for all } \lambda \in\left(\lambda_{p},+\infty\right), \\
& \psi_{1}^{i}(0)<|\hat{u}(q)| \quad \text { iff } \lambda \in \Delta_{q p}^{i} .
\end{aligned}
$$

ProOF. (i) follows from (7.13) and (4.10)-(4.11). Now, let $p \in 2 q \mathbf{Z}$; i.e., $n_{1}=1$ and $n_{2}$ even. We consider the positive measures on $\mathbf{T}$ :

$$
\mu_{1}(d s)=\lambda \hat{w}(q) g^{\prime}\{\lambda \hat{w}(q)|\hat{u}(q)| 2 \cos (2 \pi s)\} d s,
$$

and

$$
\mu_{2}(d s)=\lambda \hat{w}(q) g^{\prime}\{\lambda \hat{w}(q)|\hat{u}(q)| 2 \sin (2 \pi s)\} d s .
$$

For $\lambda \searrow \lambda_{q}$ we have $|\hat{u}(q)| \rightarrow 0$ and $\lambda_{q} \hat{w}(q) g^{\prime}(0)=1$ such that

$$
\mu_{i}(d s) \rightarrow d s \text { in the weak sense, } \quad i=1,2 .
$$

For $\lambda \rightarrow \infty,(4.11)$ shows $\mu_{i}(\mathbf{T}) \rightarrow \frac{1}{2}$, but $\mu_{1}(d s) \rightarrow 0$ for all $s \neq \pm \frac{1}{4}$ and $\mu_{2}(d s) \rightarrow 0$ for all $s \neq 0, \frac{1}{2}$. By the symmetry of $\mu_{1}$ on 0 and the symmetry of $\mu_{2}$ on $\frac{1}{4}$, we get

$$
\lim _{\lambda \rightarrow \infty} \mu_{1}=\frac{1}{4}\left(\delta_{1 / 4}+\delta_{3 / 4}\right), \quad \lim _{\lambda \rightarrow \infty} \mu_{2}=\frac{1}{4}\left(\delta_{0}+\delta_{1 / 2}\right) .
$$

Now the positive functions

$$
h_{i}(\lambda)=\int\left(1+(-1)^{i} \cos \left(4 \pi n_{2} s\right)\right) \mu_{i}(d s),
$$

which by the assertion following (9.2) with $p, q$ and $n_{1}, n_{2}$ exchanged are always less than 1 , satisfy

$$
\begin{gathered}
\lim _{\lambda \backslash \lambda_{q}} h_{i}(\lambda)=1 \text { for } i=1,2, \\
\lim _{\lambda \rightarrow \infty} h_{1}(\lambda)=\frac{1}{4}\left(2-\cos \left(\frac{4 \pi n_{2}}{4}\right)-\cos \left(\frac{4 \pi n_{2} 3}{4}\right)\right)=0
\end{gathered}
$$


and

$$
\lim _{\lambda \rightarrow \infty} h_{2}(\lambda)=\frac{1}{4}\left(2+1+\cos \left(\frac{4 \pi n_{2}}{2}\right)\right)=1
$$

Therefore

$$
\Delta_{q p}^{1}=\left\{\lambda, h_{1}(\lambda)<\hat{w}(q) / \hat{w}(p)\right\} \supseteq\left(\lambda_{q p}^{1},+\infty\right) \neq \varnothing
$$

while

$$
\Delta_{q p}^{2}=\left\{\lambda, h_{2}(\lambda)<\hat{w}(q) / \hat{w}(p)<1\right\}
$$

is a bounded, possibly empty region in $\mathbf{R}^{+}$. This proves (ii). Now, the equations uniquely determining $\psi_{i}^{1}(0)$ and $\psi_{i}^{2}(0)$ in Theorem 14 , the assertion after (9.2), and the definition of $\Delta_{q p}^{i}$ yield (iii) immediately.

PROOF OF THEOREM 7. We have already seen at the beginning of this section that the branch of $p$-primary solutions does not have a secondary bifurcation in $\mathcal{F}_{p q}$. Similar calculations as in (9.3)-(9.4), with $p, q$ and $n_{1}, n_{2}$ exchanged, show that if $\frac{1}{2} \geq \hat{w}(q) / \hat{w}(p)>0$ and $p \notin \mathbf{Z} q$, then there are no secondary bifurcation from the $q$-primary solutions. But if $1>\hat{w}(q) / \hat{w}(p)>\frac{1}{2}$ and $p \notin \mathbf{Z} q$, then Theorem 14 and the symmetry properties of $\phi^{1}, \phi^{2}$ give us the existence of eight nondegenerated $\left(\left|\hat{v}_{i}(p)\right| \neq 0 \neq\left|\hat{v}_{i}(q)\right|\right)$ fixed points

$$
\left( \pm\left|\hat{v}_{1}(p)\right|, \pm\left|\hat{v}_{1}(q)\right|\right) \text { and }\left( \pm\left|\hat{v}_{2}(p)\right|, \pm\left|\hat{v}_{2}(q)\right|\right)
$$

of $\phi^{1}$ and $\phi^{2}$, respectively, which branch from the fixed points $(0, \pm|\hat{u}(q)|)$. The fixed points (9.26) establish the secondary solutions $v_{1}$ and $v_{2}$ of (7.7)-(7.8) with $\tau_{i}=$ $j_{i}=k_{i}=0, i=1,2$. Rotating these solutions $v_{i}$ by $r_{0} \cdot\left(\tau+j_{i}^{\prime} n_{2}+k_{i}^{\prime} n_{1}\right) / n_{1} n_{2}\left(r_{0} r_{0}\right)$ with

$$
j_{i}^{\prime} n_{2} \equiv j_{i} \bmod n_{1} \quad \text { and } \quad k_{i}^{\prime} n_{1} \equiv k_{i} \bmod n_{2}, \quad i=1,2,
$$

it is easily proved by the invariance of the set of solutions of (2.1) under rotations in $\mathbf{T}^{d}$ that $v_{1}$ and $v_{2}$ given in (7.7)-(7.8) are indeed secondary solutions by any choice of the parameters.

In the same way, part (iv) of Theorem 7 follows from the results about $\Delta_{q p}^{1}$ in Theorem 14.

Let us conclude with some remarks about the stability of the solutions. The fact following (9.2) proves that the $p$-primary solutions are $p$-stable and stable with respect to all directions $\hat{u}(q) \in \mathbf{C}$. Similarly, the $q$-primary solutions are $q$-stable. In the case $p \notin q \mathbf{Z}$ they are also $p$-stable if and only if $\lambda \in \Delta_{q p} \supseteq\left(\lambda_{q p},+\infty\right)$, i.e., if $1>\hat{w}(q) / \hat{w}(p)>\frac{1}{2}$ and $h_{1}(\lambda)=h_{2}(\lambda)<\hat{w}(q) / \hat{w}(p)$. If, however, $p \in 2 \mathbf{Z} q$, then one can show that the $q$-primary solutions are stable with respect to all directions $\hat{u}(p) \in \mathbf{C}$ only if $\lambda \in \Delta_{q p}^{1} \cap \Delta_{q p}^{2}$, which is bounded in $\mathbf{R}^{+}$. It is hyperbolic or critical otherwise. If, for $\lambda \in \Delta_{q p}^{i}$, we denote by $\left(\left|\hat{v}_{i}(p)\right|,\left|\hat{v}_{i}(q)\right|\right)$ that fixed point of $\phi^{i}$ in $(0, \infty)^{2}$ with $\left|\hat{v}_{i}(q)\right|$ maximal, then we have a hyperbolic or critical fixed point of $\phi^{i}$, which also gives hyperbolic or critical secondary solutions $v_{i}, i=1,2$, by (7.7)-(7.8).

\section{REFERENCES}

0. E. Bienenstock, Cooperation and competition in C.N.S. development: a unified approach, Synergetics of the Brain (Proc. Sympos., at Schloss Elmau, 1983, E. Basar et al., eds.), Berlin and New York.

1. O. Diekmann and H. G. Kaper, On the bounded solutions of a nonlinear convolution equation, Nonlinear Anal. 2 (1978), 721-737. 
2. Th. Eisele and R. S. Ellis, Symmetry breaking and random waves for magnetic systems on a circle, Z. Wahrsch. Verw. Gebiete 63 (1983), 297-348.

3. _ The generalized Curie-Weiss model and the d-body ferromagnetic circle (to appear).

4. R. S. Ellis, J. L. Monroe and C. M. Newman, The GHS and other correlation inequalities for a class of even ferromagnets, Comm. Math. Phys. 46 (1976), 167-182.

5. G. B. Ermentrout and J. D. Cowan, Large scale spatially organized activity in neural nets, SIAM J. Appl. Math. 38 (1980), 1-21.

6. __ Secondary bifurcation in neuronal nets, SIAM J. Appl. Math. 39 (1980), 323-340.

7. D. J. Gates and O. Penrose, The van der Waals limit for classical systems. I. A variational principle, Comm. Math. Phys. 15 (1969), 255-276.

8. D. O. Hebb, The organization of behavior, Wiley, New York, 1949.

9. U. an der Heiden, Analysis of neural networks, Lecture Notes in Biomathematics, vol. 35, Springer-Verlag, Berlin and New York, 1980.

10. J. L. van Hemmen, A. C. D. van Enter and J. Canisius, On a classical spin class model, Z. Phys. B 50 (1983), 311-336.

11. G. Iooss and D. D. Joseph, Elementary stability and bifurcation theory, Springer-Verlag, New York and Berlin, 1980.

12. O. E. Lanford, Entropy and equilibrium states in classical statistical mechanics, Statistical Mechanics and Mathematical Problems (Battelle Seattle, 1971), Lecture Notes in Phys., vol. 20, Springer-Verlag, 1973, pp. 1-113.

13. Ch. van der Malsburg, Development of ocularity domains and growth behavior of axon terminals, Biol. Cybernet. 32 (1979), 49-62.

14. J. Palis and W. de Melo, Geometric theory of dynamical systems, Springer-Verlag, New York and Berlin, 1982.

15. G. Ruget, About nucleation, preprint, 1982.

16. M. Schatzman, Spatial structuration in a model in neurophysiology, preprint, 1982.

17. S. Smale, The mathematics of time, Springer-Verlag, New York and Berlin, 1980.

18. N. V. Swindale, A model for the formation of ocular dominance stripes, Proc. Roy Soc. London Ser. B 208 (1980), 243-264.

19. 215 (1982), 211-230.

20. C. L. Thompson, Mathematical statistical mechanics, Princeton Univ. Press, Princeton, N. J., 1972.

21. A. Vanderbauwhede, Local bifurcation and symmetry, Pitman, Boston, Mass., 1982.

22. D. J. Willshaw and Ch. van der Malsburg, How patterned neural connections can be set up by self-organization, Proc. Roy Soc. London Ser. B 194 (1976), 431-445.

23. F. Comets, Tunnelling and nucleation for a local mean field model, Ann. Inst. H. Poincaré (to appear).

24. P. Deufhhard, B. Fiedler and P. Kunkel, Efficient numerical path following beyond critical points, Preprint 278, SFB 123, Heidelberg, 1984.

25. Th. Eisele, Equilibrium and nonequilibrium theory of a geometric longrange spinglass, Proc. Summer School Les Houches 1984 on Critical Phenomena, Random Systems and Gauge Theories.

Université de Paris Sud, Centre D'Orsay, BÂtiment de Mathématique 425, F-91405 ORSAY CEDEX, FRANCE (Current address of F. Comets)

INSTITUT FÜR ANGEWANDTE MATHEMATIK, UNIVERTSITÄT HEIDELBERG, IM NEUENHEIMER FELD 294, D-6900 HeIDELBERG, GERMANY

Université Claude Bernard, Département de Mathématiques, F-69622 Villeurbanne Cedex, France (Current address of M. Schatzman)

Current address (Th. Eisele): Courant Institute of Mathematical Sciences, New York University, New York, New York 10012 